\title{
Parity violation in quasifree electron scattering off the deuteron*
}

\author{
G. Küster ${ }^{\dagger}$ and H. Arenhövel \\ Institut für Kernphysik, Johannes Gutenberg-Universität, D-55099 Mainz, Germany
}

(January 3, 2018)

\begin{abstract}
For deuteron electroweak disintegration, parity violating effects are investigated which arise from the interference of $\gamma$ and $Z$ exchange as well as from the hadronic sector via a small parity violating component in the deuteron. The general formalism for the differential cross section and polarization observables of electromagnetic deuteron disintegration is extended to incorporate parity violating contributions. Formal expressions for the additional structure functions are derived. Results are presented for these parity violating structure functions for quasifree kinematics neglecting final state interaction and two-body effects. Both types of parity violating contributions to the asymmetry of the inclusive reaction with respect to longitudinally polarized electrons are evaluated. The one from parity violating deuteron components is negligible over the whole range of momentum transfers considered.
\end{abstract}

PACS numbers: 12.15.Ji, 13.60.-r, 24.70.+s, 24.80.+y, 25.30.Fj

\section{INTRODUCTION}

After the discovery of parity violation in weak processes in 1957, their manifestation in electromagnetic and strong processes has been subject of many detailed investigations [1 $[$ [6]. The recent interest in studying parity violation by electroweak interference is motivated by the possibility to investigate the so-called strangeness or better $s \bar{s}$-content of the nucleon, a quantity of particular interest with respect to the nucleon spin structure functions as measured in deep inelastic scattering [7 9]. In fact, several experiments to measure parity violation in electron scattering off hydrogen and deuterium are presently underway 10 13]. In these experiments, deuterium serves as a neutron target and for this reason quasifree kinematics is preferred in order to minimize possible interaction effects.

Parity violation in inelastic electron deuteron scattering has been studied before theoretically by Hwang et al. [14, 15 ] in the low energy and momentum transfer domain and by Hadjimichael et al. [16] for a larger kinematical range, in particular for quasifree kinematics at high momentum transfers. Relativistic contributions have been considered recently by Poulis 17,18]. Furthermore, during completion of this work another calculation by Mosconi and Ricci appeared [19]. While in 15] the parity violation by electroweak interference as well as through parity violating nuclear components has been considered, the latter has been neglected throughout in the more recent evaluations [16 19], although the authors of [16] remark that such effects should be investigated in order to see for which kinematical situations they can indeed be neglected or have to be included. This could be of relevance for the SAMPLE experiment [13] which will measure the strange magnetic form factor $G_{M}^{(s)}$ at low momentum transfer.

It is, therefore, the purpose of this study to investigate parity violation in deuteron disintegration by electrons in the quasifree region for energy and momentum transfers far beyond the ones studied in [15] by considering simultaneously both possibilities, i.e., on one hand parity violation in the deuteron via a parity violating part in the $N N$ potential, and on the other hand in the scattering process itself through the electroweak interference from virtual photon and $Z$ exchange.

The presence of a parity violating $N N$ potential $V^{p n c}$ has the consequence that the nuclear states are no longer states of good parity, i.e., besides the dominant state $\left|J^{\pi}\right\rangle$ of angular momentum $J$ and parity $\pi$ there will be a small admixture of opposite parity $\left|J^{-\pi}\right\rangle$, with an amplitude $\mathcal{F}$ of the order $10^{-6}$ [20]. In the case of the deuteron, the admixture of opposite parity components must result in a $P$-wave, given the possible spin states and the total angular momentum. For the calculation of these $P$-wave components we will use for $V^{p n c}$ the one-boson-exchange model of Desplanques, Donoghue and Holstein [21]. Based on the quark model and $S U(6)_{w}$ symmetry, they made predictions for all meson-nucleon couplings from both charged and neutral current pieces of the weak Hamiltonian.

This paper is organized as follows: In Sect. II we extend the basic formalism for describing pure electromagnetic electrodisintegration of the deuteron to the electroweak case, and introduce additional parity violating structure

\footnotetext{
* Supported by the Deutsche Forschungsgemeinschaft (SFB 201)

${ }^{\dagger}$ Present address: Deutsches Krebsforschungszentrum, Forschungsschwerpunkt 05, D-69120 Heidelberg, Germany
} 
functions. In Sect. III the parity violating $N N$ potential is introduced and the parity violating components to the deuteron wave function are calculated. Furthermore, we show a few of the resulting new pnc (parity nonconserved) structure functions for the quasifree case. The pnc structure functions corresponding to the second mechanism of parity violation, i.e., electroweak interference, are considered in Sect. IV. The quantity of experimental interest, the longitudinal asymmetry of the inclusive reaction, is investigated for both cases in Sect. $\mathrm{\nabla}$. Finally, we summarize and give some conclusions in Sect. VI.

\section{FORMALISM FOR ELECTROWEAK DISINTEGRATION}

In this section we briefly present the basic formalism for the electroweak disintegration of the deuteron. We follow closely the formalism and notation for electrodisintegration including polarization effects as outlined in Ref. [22] and extend it to the electroweak case.

The general expression for the cross section including beam and target polarization is given by

$$
d \sigma_{f i}=(2 \pi)^{-5} \delta^{(4)}\left(p_{n}+p_{p}-q-P_{d}\right) \operatorname{tr}\left(\mathcal{M}_{f i}^{\dagger} \mathcal{M}_{f i} \hat{\rho}^{e} \hat{\rho}^{d}\right) \frac{m_{e}^{2} d^{3} k_{2}}{4 k_{10} k_{20}} \frac{M^{2}}{M_{d}} \frac{d^{3} p_{n}}{E_{n}} \frac{d^{3} p_{p}}{E_{p}} .
$$

The momenta of the initial and the scattered electrons are denoted by $k_{1}$ and $k_{2}$, respectively, while $q_{\mu}^{2}=q_{0}^{2}-\vec{q}^{2}$ is the four momentum transfer squared $\left(q=k_{1}-k_{2}\right)$. The deuteron and final proton and neutron momenta are denoted by $P_{d}$ and $p_{p / n}$ and their masses by $M$ and $M_{d}$, respectively. The density matrices $\hat{\rho}^{e}$ and $\hat{\rho}^{d}$ describe possible beam and target polarization. Covariant normalization has been assumed, i.e., $(2 \pi)^{3} E / m$ for fermions and $(2 \pi)^{3} 2 E$ for bosons.

The amplitude $\mathcal{M}_{f i}$ contains in the lowest order contributions from both virtual $\gamma$ and $Z$ exchange (see Fig. 1) with the latter naturally being strongly suppressed since we restrict ourselves to the low momentum transfer region $\left(-q_{\mu}^{2} \ll M_{Z}^{2}\right)$. The invariant matrix element in the one-boson-exchange approximation thus contains two contributions 23]

$$
\mathcal{M}_{f i}=\frac{e^{2}}{q_{\mu}^{2}} j^{(\gamma) \mu} J_{f i, \mu}^{(\gamma)}+\sqrt{2} G_{F} j^{(Z) \mu} J_{f i, \mu}^{(Z)} .
$$

Here and in the following, the superscripts $\gamma$ and $Z$ indicate the electromagnetic and weak neutral current contributions. The lepton and hadron currents are denoted by $j_{\mu}^{(\gamma / Z)}$ and $J_{f i, \mu}^{(\gamma / Z)}$, respectively. Furthermore, e denotes the elementary charge with $\alpha=e^{2} / 4 \pi$ as fine structure constant, and the weak Fermi coupling constant $G_{F}$ is given by

$$
\frac{G_{F}}{\sqrt{2}}=\frac{g^{2}}{8 \cos ^{2} \theta_{W} M_{Z}^{2}},
$$

where $g$ denotes the electroweak coupling constant, $\theta_{W}$ the Weinberg angle, and $e=g \sin \theta_{W}$. In the following we use $\sin ^{2} \theta_{W}=0.232$. The lepton currents are defined by

$$
\begin{aligned}
& j^{(\gamma) \mu}=\bar{u}\left(k_{2}\right) \gamma^{\mu} u\left(k_{1}\right), \\
& j^{(Z) \mu}=\bar{u}\left(k_{2}\right) \gamma^{\mu}\left(g_{v}+g_{a} \gamma_{5}\right) u\left(k_{1}\right),
\end{aligned}
$$

with

$$
\begin{aligned}
& g_{v}=-\frac{1}{2}+2 \sin ^{2} \theta_{W}, \\
& g_{a}=\frac{1}{2} .
\end{aligned}
$$

The hadronic currents are given later in Sect. IV. Note, that our expressions for the neutral currents contain an additional factor $1 / 2$ compared to Ref. [23].

Allowing for longitudinal electron polarization of degree $h$, one finds the well known expression [6]

$$
\begin{aligned}
m_{e}^{2} \operatorname{tr}\left(\mathcal{M}_{f i}^{\dagger} \mathcal{M}_{f i} \hat{\rho}^{e} \hat{\rho}^{d}\right)= & \left(\frac{e^{2}}{q_{\mu}^{2}}\right)^{2} \eta_{\mu \nu}^{\gamma \gamma}(h) W_{f i}^{\gamma \gamma, \mu \nu}\left(\hat{\rho}^{d}\right)+2 G_{F}^{2} \eta_{\mu \nu}^{Z Z}(h) W_{f i}^{Z Z, \mu \nu}\left(\hat{\rho}^{d}\right) \\
& +\sqrt{2} G_{F} \frac{e^{2}}{q_{\mu}^{2}} \eta_{\mu \nu}^{\gamma Z}(h)\left(W_{f i}^{\gamma Z, \mu \nu}\left(\hat{\rho}^{d}\right)+W_{f i}^{Z \gamma, \mu \nu}\left(\hat{\rho}^{d}\right)\right)
\end{aligned}
$$


where the various lepton tensors are given by

$$
\begin{aligned}
\eta_{\mu \nu}^{\gamma \gamma}(h) & =\eta_{\mu \nu}^{v v}(h), \\
\eta_{\mu \nu}^{\gamma Z}(h) & =g_{v} \eta_{\mu \nu}^{v v}(h)+g_{a} \eta_{\mu \nu}^{v a}(h), \\
\eta_{\mu \nu}^{Z Z}(h) & =\left(g_{v}^{2}+g_{a}^{2}\right) \eta_{\mu \nu}^{v v}(h)+2 g_{v} g_{a} \eta_{\mu \nu}^{v a}(h) .
\end{aligned}
$$

Since the $Z$ couples to both the vector and the axial vector current (with couplings $g_{v}$ and $g_{a}$ ), one has, compared to the pure electromagnetic case, now two types of lepton tensors $\eta_{\mu \nu}^{v v}$ and $\eta_{\mu \nu}^{v a}$, where the latter arises from the interference of the vector with the axial vector current,

$$
\begin{aligned}
& \eta_{\mu \nu}^{v v}(h)=\eta_{\mu \nu}^{0}+h \eta_{\mu \nu}^{\prime}, \\
& \eta_{\mu \nu}^{v a}(h)=\eta_{\mu \nu}^{\prime}+h \eta_{\mu \nu}^{0} .
\end{aligned}
$$

In the high energy limit, i.e., electron mass $m_{e}=0$, one has

$$
\begin{aligned}
\eta_{\mu \nu}^{0} & =\left(k_{1 \mu} k_{2 \nu}+k_{2 \mu} k_{1 \nu}\right)-g_{\mu \nu} k_{1} \cdot k_{2} \\
& =\frac{1}{2}\left(k_{\mu} k_{\nu}-q_{\mu} q_{\nu}+g_{\mu \nu} q_{\rho}^{2}\right) \\
\eta_{\mu \nu}^{\prime} & =i \varepsilon_{\mu \nu \alpha \beta} k_{1}^{\alpha} k_{2}^{\beta} \\
& =\frac{i}{2} \varepsilon_{\mu \nu \alpha \beta} k^{\alpha} q^{\beta}
\end{aligned}
$$

where $k=k_{1}+k_{2}$. The hadronic tensors appearing in (8) are given by the electromagnetic and weak current matrix elements, for example,

$$
W_{f i}^{\gamma \gamma, \mu \nu}\left(\hat{\rho}^{d}\right)=\operatorname{tr}\left(J_{f i}^{(\gamma) \mu \dagger} J_{f i}^{(\gamma) \nu} \hat{\rho}^{d}\right),
$$

where the trace refers to the deuteron spin quantum numbers.

Proceeding as in the electromagnetic case, one obtains in analogy to the pure electromagnetic process the following expression for the differential cross section including both beam and target polarization (for details see [24])

$$
\begin{aligned}
\frac{d^{3} \sigma^{\gamma+Z}}{d k_{2}^{l a b} d \Omega_{k_{2}}^{l a b} d \Omega_{n p}^{c . m .} .}=\frac{\alpha}{2 \pi^{2}} \frac{k_{2}^{l a b}}{k_{1}^{l a b} q_{\mu}^{4}} & \sum_{\lambda \lambda^{\prime} s m_{s} m_{d} m_{d}^{\prime}} \rho_{m_{d} m_{d}^{\prime}}^{d}\left(\rho_{\lambda \lambda^{\prime}}^{v v} T_{s m_{s} \lambda m_{d}}^{\gamma} T_{s m_{s} \lambda^{\prime} m_{d}^{\prime}}^{\gamma *}\right. \\
& \left.+\frac{G_{F}}{\sqrt{2}} \frac{q_{\mu}^{2}}{\pi \alpha}\left(g_{v} \rho_{\lambda \lambda^{\prime}}^{v v}+g_{a} \rho_{\lambda \lambda^{\prime}}^{v a}\right) \Re e\left[T_{s m_{s} \lambda m_{d}}^{\gamma} T_{s m_{s} \lambda^{\prime} m_{d}^{\prime}}^{Z{ }^{*}}\right]\right),
\end{aligned}
$$

where the terms quadratic in the weak amplitude $\left(T_{\mu}^{Z *} T_{\nu}^{Z}\right)$ have been omitted since they are of order $\mathcal{O}\left(q_{\mu}^{4} / M_{Z}^{4}\right)$ compared to the electromagnetic process. The direction of the outgoing proton in the final $n p$ c.m. system is denoted by $\Omega_{n p}$. One finds for the spherical components of the two types of virtual boson density matrices

$$
\begin{aligned}
& \rho_{\lambda \lambda^{\prime}}^{v v}=\rho_{\lambda \lambda^{\prime}}^{0}+h \rho_{\lambda \lambda^{\prime}}^{\prime}, \\
& \rho_{\lambda \lambda^{\prime}}^{v a}=\rho_{\lambda \lambda^{\prime}}^{\prime}+h \rho_{\lambda \lambda^{\prime}}^{0} .
\end{aligned}
$$

They obey the symmetry relations

$$
\begin{aligned}
\rho_{\lambda \lambda^{\prime}}^{v v / v a} & =\rho_{\lambda^{\prime} \lambda}^{v v / v a}, \\
\rho_{-\lambda-\lambda^{\prime}}^{0} & =(-)^{\lambda+\lambda^{\prime}} \rho_{\lambda \lambda^{\prime}}^{0}, \\
\rho_{-\lambda-\lambda^{\prime}}^{\prime} & =(-)^{\lambda+\lambda^{\prime}+1} \rho_{\lambda \lambda^{\prime}}^{\prime} .
\end{aligned}
$$

The nonvanishing components are

$$
\begin{aligned}
& \rho_{L}=\rho_{00}^{0}=-\beta^{2} q_{\nu}^{2} \frac{\xi^{2}}{2 \eta}, \rho_{T}=\rho_{11}^{0}=-\frac{1}{2} q_{\nu}^{2}\left(1+\frac{\xi}{2 \eta}\right), \\
& \rho_{L T}=\rho_{01}^{0}=-\beta q_{\nu}^{2} \frac{\xi}{\eta} \sqrt{\frac{\eta+\xi}{8}}, \quad \rho_{T T}=\rho_{-11}^{0}=q_{\nu}^{2} \frac{\xi}{4 \eta} \\
& \rho_{L T}^{\prime}=\rho_{01}^{\prime}=-\frac{1}{2} \beta \frac{q_{\nu}^{2}}{\sqrt{2 \eta}} \xi, \quad \rho_{T}^{\prime}=\rho_{11}^{\prime}=-\frac{1}{2} q_{\nu}^{2} \sqrt{\frac{\eta+\xi}{\eta}}
\end{aligned}
$$


with

$$
\beta=\frac{\left|\vec{q}^{l a b}\right|}{\left|\vec{q}^{c}\right|}, \quad \xi=-\frac{q_{\nu}^{2}}{\left|\vec{q}^{l a b}\right|^{2}}, \quad \eta=\tan ^{2} \frac{\theta_{e}}{2},
$$

where $\beta$ expresses the boost from the lab system to the frame in which the hadronic tensor is evaluated and $\vec{q}^{c}$ denotes the momentum transfer in this frame. In this work, we take the c.m. frame of the final $n p$ state for the evaluation, and the momentum transfer in this frame will be denoted by $\vec{q}$ throughout. In order to make contact to the kinematic functions $v_{\alpha^{(\prime)}}$ in the review of Musolf et al. [6], we note the simple relation

$$
\rho_{\alpha}^{(\prime)}=-\frac{q_{\mu}^{2}}{2 \eta} v_{\alpha^{(\prime)}}
$$

where $\alpha=L, T, L T, T T$.

In Eq. (17), the $T$-matrices were introduced, which are related to the electromagnetic and neutral current matrix elements between intrinsic states by

$$
\begin{aligned}
& T_{s m_{s} \lambda m_{d}}^{\gamma}=-\pi \sqrt{2 \alpha p_{n p} E E_{d} / M_{d}}\left\langle s m_{s}\left|J_{\lambda}^{\gamma}(\vec{q}, \vec{Q})\right| m_{d}\right\rangle, \\
& T_{s m_{s} \lambda m_{d}}^{Z}=-\pi \sqrt{2 \alpha p_{n p} E E_{d} / M_{d}}\left\langle s m_{s}\left|J_{\lambda}^{Z}(\vec{q}, \vec{Q})\right| m_{d}\right\rangle,
\end{aligned}
$$

where $\lambda= \pm$ refer to the transverse current components (with respect to $\vec{q}$ ), while the $\lambda=0$ component is given by

$$
\begin{aligned}
J_{0} & =-\frac{|\vec{q}|^{2}}{q_{\mu}^{2}}\left(\rho-\frac{\omega}{|\vec{q}|^{2}} \vec{q} \cdot \vec{J}\right) \\
& =\rho-\frac{\omega}{q_{\mu}^{2}}(\omega \rho-\vec{q} \cdot \vec{J}),
\end{aligned}
$$

which reduces to the charge density $\rho$ for a conserved current. Furthermore, $E_{d}$ and $E$ denote the deuteron and nucleon energies in the $n p$ c.m. frame, respectively. Here, the c.m. motion of the initial and final hadronic states with c.m. momenta $\vec{P}_{i}$ and $\vec{P}_{f}$, respectively, has been eliminated and we have switched to noncovariant normalization. One should note that the intrinsic current operators depend also on $\vec{Q}=\vec{P}_{i}+\vec{P}_{f}$.

The final intrinsic state is characterized by the relative $n p$-momentum $\vec{p}_{n p}$, the spin $s$ and its projection $m_{s}$ for which the direction of $\vec{p}_{n p}$ is chosen as quantization axis, whereas for the initial deuteron state the momentum $\vec{q}$ of the virtual boson is chosen as quantization axis. The direction of $\vec{p}_{n p}$ is described by the angles $\theta$ and $\phi$ with respect to a coordinate system whose $z$-axis is chosen parallel to $\vec{q}$ and the $y$-axis parallel to $\vec{k}_{1} \times \vec{k}_{2}$ (for details see Fig. 2 and $[22])$.

Then the $\phi$-dependence of the $T$-matrices can be separated so that the reduced $t$-matrices depend on $\theta$ and $|\vec{q}|$ only

$$
T_{s m_{s} \lambda m_{d}}^{\gamma / Z}(\theta, \phi)=e^{i\left(\lambda+m_{d}\right) \phi} t_{s m_{s} \lambda m_{d}}^{\gamma / Z}(\theta) .
$$

The electromagnetic current matrix element can be split into a leading, parity conserving part $\left(\gamma_{v}\right)$ and a very small contribution ( $\gamma_{a}$, analogue to an axial current) from parity violating components of the wave functions

$$
t_{s m_{s} \lambda m_{d}}^{\gamma}(\theta)=t_{s m_{s} \lambda m_{d}}^{\gamma_{v}}(\theta)+t_{s m_{s} \lambda m_{d}}^{\gamma_{a}}(\theta) .
$$

For the parity conserved part, the transformation properties under space and time inversion lead to the following symmetry property for the reduced $t$-matrices

$$
t_{s-m_{s}-\lambda-m_{d}}^{\gamma_{v}}=(-)^{1+s+m_{s}+\lambda+m_{d}} t_{s m_{s} \lambda m_{d}}^{\gamma_{v}},
$$

while for the parity violating part one finds

$$
t_{s-m_{s}-\lambda-m_{d}}^{\gamma_{a}}=(-)^{s+m_{s}+\lambda+m_{d}} t_{s m_{s} \lambda m_{d}}^{\gamma_{a}} .
$$

Just as for the leptonic current, one has two contributions for the weak hadronic current, namely a vector and an axial vector piece

$$
t_{s m_{s} \lambda m_{d}}^{Z}(\theta)=t_{s m_{s} \lambda m_{d}}^{Z_{v}}(\theta)+t_{s m_{s} \lambda m_{d}}^{Z_{a}}(\theta) .
$$


The reduced $t$-matrices $t_{s m_{s} \lambda m_{d}}^{Z_{\text {a }}}$ have the same symmetry properties as in (33) for $v$ and in (34) for $a$.

Now one can proceed exactly as in 22] by inserting the deuteron density matrix, which is characterized by vector and tensor polarization parameters $P_{1}^{d}$ and $P_{2}^{d}$, respectively, and by the angles $\theta_{d}$ and $\phi_{d}$, describing the direction of the orientation axis $\hat{d}$ of the polarized deuteron target with respect to the coordinate system associated with the three-momentum transfer $\vec{q}$ (see [22] for details), and exploiting the symmetry properties of the boson density matrices and the reduced $t$-matrices. It is useful, in order to distinguish the contributions according to their parity properties, to introduce the following two index sets

$$
\begin{aligned}
& \mathcal{S}_{v}:=\left\{\gamma_{v}, Z_{v}\right\}, \\
& \mathcal{S}_{a}:=\left\{\gamma_{a}, Z_{a}\right\},
\end{aligned}
$$

because, as will be apparent below, the contributions to the cross section from the same set have formally the same type of structure functions.

In Ref. 22, all observables had been classified according to their parity properties, i.e., a set $A$ for the scalar and a set $B$ for the pseudoscalar observables. To each set belongs a corresponding set of structure functions. Having now two types of $t$-matrices ( $v$ - and $a$-type) it is obvious that the combinations $v-v$ and $a$ - $a$ lead to the same type of structure functions as in [22], whereas for the $v-a$ combinations the role of the $A$ - and $B$-type observables is interchanged. Keeping this in mind, one finds the following compact expression for the differential cross section

$$
\begin{aligned}
S\left(h, P_{1}^{d}, P_{2}^{d}\right)= & \frac{d^{3} \sigma^{\gamma+Z}}{d k_{2}^{l a b} d \Omega_{k_{2}}^{\text {lab }} d \Omega_{n p}^{c . m .}} \\
= & \bar{S}\left(\gamma_{v} ; P_{1}^{d}, P_{2}^{d}\right)+\bar{S}\left(\gamma_{a} ; P_{1}^{d}, P_{2}^{d}\right) \\
& +a_{v}\left(\bar{S}\left(Z_{v} ; P_{1}^{d}, P_{2}^{d}\right)+\bar{S}\left(Z_{a} ; P_{1}^{d}, P_{2}^{d}\right)\right) \\
& +a_{a}\left(\bar{S}^{\prime}\left(Z_{v} ; P_{1}^{d}, P_{2}^{d}\right)+\bar{S}^{\prime}\left(Z_{a} ; P_{1}^{d}, P_{2}^{d}\right)\right) \\
& +h\left[\bar{S}^{\prime}\left(\gamma_{v} ; P_{1}^{d}, P_{2}^{d}\right)+\bar{S}^{\prime}\left(\gamma_{a} ; P_{1}^{d}, P_{2}^{d}\right)\right. \\
& +a_{v}\left(\bar{S}^{\prime}\left(Z_{v} ; P_{1}^{d}, P_{2}^{d}\right)+\bar{S}^{\prime}\left(Z_{a} ; P_{1}^{d}, P_{2}^{d}\right)\right) \\
& \left.+a_{a}\left(\bar{S}\left(Z_{v} ; P_{1}^{d}, P_{2}^{d}\right)+\bar{S}\left(Z_{a} ; P_{1}^{d}, P_{2}^{d}\right)\right)\right],
\end{aligned}
$$

where we have defined

$$
a_{v / a}=\frac{G_{F}}{\sqrt{2}} \frac{q_{\mu}^{2}}{\pi \alpha} g_{v / a} .
$$

The abnormal parity admixture in the deuteron has been considered for photon exchange only. Here, we have introduced for $C \in \mathcal{S}_{v} \cup \mathcal{S}_{a}$, where $C$ characterizes the different current contributions,

$$
\begin{aligned}
\bar{S}\left(C ; P_{1}^{d}, P_{2}^{d}\right)= & c \sum_{I=0}^{2} P_{I}^{d} \sum_{M=0}^{I}\left\{\left(\rho_{L} f_{L}^{I M, C}+\rho_{T} f_{T}^{I M, C}+\rho_{L T} f_{L T}^{I M+, C} \cos \phi\right.\right. \\
& \left.+\rho_{T T} f_{T T}^{I M+, C} \cos 2 \phi\right) \cos \left(M \tilde{\phi}-\bar{\delta}_{I}^{C} \frac{\pi}{2}\right) \\
& \left.-\left(\rho_{L T} f_{L T}^{I M-, C} \sin \phi+\rho_{T T} f_{T T}^{I M-, C} \sin 2 \phi\right) \sin \left(M \tilde{\phi}-\bar{\delta}_{I}^{C} \frac{\pi}{2}\right)\right\} d_{M 0}^{I}\left(\theta_{d}\right), \\
\bar{S}^{\prime}\left(C ; P_{1}^{d}, P_{2}^{d}\right)= & c \sum_{I=0}^{2} P_{I}^{d} \sum_{M=0}^{I}\left\{\left(\rho_{T}^{\prime} f_{T}^{I M, C}+\rho_{L T}^{\prime} f_{L T}^{I M-, C} \cos \phi\right) \sin \left(M \tilde{\phi}-\bar{\delta}_{I}^{C} \frac{\pi}{2}\right)\right. \\
& \left.+\rho_{L T}^{\prime} f_{L T}^{\prime I M+, C} \sin \phi \cos \left(M \tilde{\phi}-\bar{\delta}_{I}^{C} \frac{\pi}{2}\right)\right\} d_{M 0}^{I}\left(\theta_{d}\right),
\end{aligned}
$$

with

$$
c=\frac{\alpha}{6 \pi^{2}} \frac{k_{2}^{l a b}}{k_{1}^{l a b} q_{\nu}^{4}}=-\frac{\eta}{2 q_{\nu}^{2}} \frac{\sigma_{M}}{3 \pi^{2} \alpha},
$$

where $\sigma_{M}$ denotes the Mott cross section, $\tilde{\phi}=\phi-\phi_{d}$ and 


$$
\bar{\delta}_{I}^{C}:=\left\{\begin{array}{cc}
\delta_{I 1} & \text { for } C \in \mathcal{S}_{v} \\
1-\delta_{I 1} & \text { for } C \in \mathcal{S}_{a}
\end{array}\right\}
$$

For $C \in \mathcal{S}_{v}$, the structure functions correspond to those of the $A$-type observables in [22] while for $C \in \mathcal{S}_{a}$, they correspond to the ones of the $B$-type observables as already mentioned above.

The various structure functions which appear in (40) and (41) are defined by

$$
\begin{aligned}
f_{L}^{I M, C} & =\frac{2}{1+\delta_{M 0}} \Re e\left[i^{\bar{\delta}_{I}^{C}} v_{00 I M}^{C}\right], \\
f_{T}^{I M, C} & =\frac{4}{1+\delta_{M 0}} \Re e\left[i^{\bar{\delta}_{I}^{C}} v_{11 I M}^{C}\right], \\
f_{L T}^{I M \pm, C} & =\frac{4}{1+\delta_{M 0}} \Re e\left[i^{\bar{\delta}_{I}^{C}}\left(v_{01 I M}^{C} \pm(-)^{I+M+\delta_{C}} v_{01 I-M}^{C}\right)\right], \\
f_{T T}^{I M \pm, C} & =\frac{2}{1+\delta_{M 0}} \Re e\left[i^{\bar{\delta}_{I}^{C}}\left(v_{-11 I M}^{C} \pm(-)^{I+M+\delta_{C}} v_{-11 I-M}^{C}\right)\right], \\
f_{T}^{\prime I M, C} & =\frac{4}{1+\delta_{M 0}} \Re e\left[i^{1+\bar{\delta}_{I}^{C}} v_{11 I M}^{C}\right], \\
f_{L T}^{\prime I M \pm, C} & =\frac{4}{1+\delta_{M 0}} \Re e\left[i^{1+\bar{\delta}_{I}^{C}}\left(v_{01 I M}^{C} \pm(-)^{I+M+\delta_{C}} v_{01 I-M}^{C}\right)\right],
\end{aligned}
$$

where $M \geq 0$ and $C \in \mathcal{S}_{v} \cup \mathcal{S}_{a}$. Furthermore, we have introduced

$$
\delta_{C}:=\left\{\begin{array}{ll}
0 & \text { for } C \in \mathcal{S}_{v} \\
1 & \text { for } C \in \mathcal{S}_{a}
\end{array}\right\} .
$$

For completeness we list for all polarization observables the corresponding structure functions in the Appendix.

Note that some of the structure functions vanish identically. First of all, for those with $M=0$ one finds from the above definitions

$$
\begin{aligned}
& f_{\alpha}^{I 0-, C}=0 \text { for }(-)^{I+\delta_{C}}=1, \\
& f_{\alpha}^{I 0+, C}=0 \text { for }(-)^{I+\delta_{C}}=-1,
\end{aligned}
$$

for $\alpha=L, T, L T, T T$. Additional vanishing structure functions follow from the symmetry properties of $v_{\lambda \mu I M}^{C}$ discussed below.

The quantities $v_{\lambda \mu I M}^{C}$ are given in terms of the $t$-matrix elements by

$$
\begin{aligned}
& v_{\lambda \mu I M}^{C}(\theta)=\frac{\hat{I} \sqrt{3}}{1+\delta_{C, \gamma_{v}}} \sum_{m m^{\prime}}(-)^{1-m}\left(\begin{array}{ccc}
1 & 1 & I \\
m & -m^{\prime} & -M
\end{array}\right) \\
& \sum_{s m_{s}}\left[\left(t_{s m_{s} \lambda m^{\prime}}^{\gamma_{v}}(\theta)\right)^{*} t_{s m_{s} \mu m}^{C}(\theta)+\left(t_{s m_{s} \lambda m^{\prime}}^{C}(\theta)\right)^{*} t_{s m_{s} \mu m}^{\gamma_{v}}(\theta)\right] .
\end{aligned}
$$

They obey two symmetry relations. The first one follows from (33) and (34)

$$
v_{-\lambda-\mu I-M}^{C}=(-)^{I-M+\lambda-\mu+\delta_{C}} v_{\lambda \mu I M}^{C},
$$

and the second one from complex conjugation

$$
\left(v_{\lambda \mu I M}^{C}\right)^{*}=(-)^{M} v_{\mu \lambda I-M}^{C} .
$$

These relations may be combined to give

$$
\left(v_{\lambda \mu I M}^{C}\right)^{*}=(-)^{I+\lambda-\mu+\delta_{C}} v_{-\mu-\lambda I M}^{C} .
$$

In particular, one finds from (55)

$$
\left(v_{\lambda \lambda I M}^{C}\right)^{*}=(-)^{M} v_{\lambda \lambda I-M}^{C},
$$

implying that $v_{\lambda \lambda I 0}^{C}$ is real, and from (56) 


$$
\left(v_{\lambda-\lambda I M}^{C}\right)^{*}=(-)^{I+\delta_{C}} v_{\lambda-\lambda I M}^{C}
$$

from which follows that $v_{\lambda-\lambda I M}^{C}$ is real or imaginary for $(-)^{I+\delta_{C}}=1$ or -1 , respectively.

The structure functions without target polarization $((I M)=(00))$ are then for $C \in \mathcal{S}_{v}$ with the notation $f_{\alpha}^{(\prime) C} \equiv$ $f_{\alpha}^{(\prime) 00+, C}$, and using (57)

$$
\begin{aligned}
f_{L}^{C}=v_{0000}^{C}, & f_{T}^{C}=2 v_{1100}^{C}, \\
f_{L T}^{C}=4 \Re e\left(v_{0100}^{C}\right), & f_{T T}^{C}=2 v_{-1100}^{C}, \\
f_{T}^{\prime C}=0, \quad & f_{L T}^{\prime C}=-4 \Im m\left(v_{0100}^{C}\right) .
\end{aligned}
$$

Analogously, one finds for the parity violating structure functions $\left(C \in \mathcal{S}_{a}\right)$ without target polarization with the notation $f_{\alpha}^{(\prime) C} \equiv f_{\alpha}^{(\prime) 00-, C}$, and using again (57)

$$
\begin{aligned}
f_{L}^{C}=0, & f_{T}^{C}=0, \\
f_{L T}^{C}=-4 \Im m\left(v_{0100}^{C}\right), & f_{T T}^{C}=-2 \Im m\left(v_{-1100}^{C}\right), \\
f_{T}^{\prime C}=-2 v_{1100}^{C}, & f_{L T}^{\prime C}=-4 \Re e\left(v_{0100}^{C}\right) .
\end{aligned}
$$

Then we find for the differential coincidence cross section without target polarization

$$
\begin{aligned}
S(h, 0,0)= & c\left\{\rho_{L}\left(f_{L}^{\gamma_{v}}+a_{v} f_{L}^{Z_{v}}\right)+\rho_{T}\left(f_{T}^{\gamma_{v}}+a_{v} f_{T}^{Z_{v}}\right)\right. \\
& +\rho_{L T}\left(\left(f_{L T}^{\gamma_{v}}+a_{v} f_{L T}^{Z_{v}}\right) \cos \phi+\left(f_{L T}^{\gamma_{a}}+a_{v} f_{L T}^{Z_{a}}\right) \sin \phi\right) \\
& +\rho_{T T}\left(\left(f_{T T}^{\gamma_{v}}+a_{v} f_{T T}^{Z_{v}}\right) \cos 2 \phi+\left(f_{T T}^{\gamma_{a}}+a_{v} f_{T T}^{Z_{a}}\right) \sin 2 \phi\right) \\
& -\rho_{T}^{\prime} a_{a} f_{T}^{\prime Z_{a}}-\rho_{L T}^{\prime} a_{a}\left(f_{L T}^{\prime Z_{a}} \cos \phi-f_{L T}^{\prime Z_{v}} \sin \phi\right) \\
+ & h\left[\rho_{L} a_{a} f_{L}^{Z_{v}}+\rho_{T} a_{a} f_{T}^{Z_{v}}+\rho_{L T} a_{a}\left(f_{L T}^{Z_{v}} \cos \phi+f_{L T}^{Z_{a}} \sin \phi\right)\right. \\
& +\rho_{T T} a_{a}\left(f_{T T}^{Z_{v}} \cos 2 \phi+f_{T T}^{Z_{a}} \sin 2 \phi\right)-\rho_{T}^{\prime}\left(f_{T}^{\prime \gamma_{a}}+a_{v} f_{T}^{\prime Z_{a}}\right) \\
& \left.\left.-\rho_{L T}^{\prime}\left(\left(f_{L T}^{\prime \gamma_{a}}+a_{v} f_{L T}^{\prime Z_{a}}\right) \cos \phi-\left(f_{L T}^{\prime \gamma_{v}}+a_{v} f_{L T}^{\prime Z_{v}}\right) \sin \phi\right)\right]\right\} .
\end{aligned}
$$

It is apparent that parity violation in the hadronic sector $\left(\gamma_{a}\right)$ and via the weak axial hadron current $\left(Z_{a}\right)$ lead to the same type of structure functions so that they always appear together in the combination $f_{\alpha}^{(\prime) \gamma_{a}}+a_{v} f_{\alpha}^{(\prime)} Z_{a}(\operatorname{see}$ [6]). Similarly, the vector contributions appear always in the form $f_{\alpha}^{(\prime) \gamma_{v}}+a_{v} f_{\alpha}^{(\prime) Z_{v}}$. We note in passing, that such an expression without the $\gamma_{a}$-contributions has also been derived in [19].

The inclusive cross section for an unpolarized target, denoted by $\Sigma(h)$, is then obtained by integration over $\Omega_{n p}$ of the outgoing nucleon

$$
\begin{aligned}
\Sigma(h)= & d^{2} \sigma^{\gamma+Z} / d k_{2}^{l a b} d \Omega_{k_{2}}^{l a b}=\int d \Omega_{n p} S(h, 0,0) \\
= & c\left\{\rho_{L}\left(F_{L}^{\gamma_{v}}+a_{v} F_{L}^{Z_{v}}\right)+\rho_{T}\left(F_{T}^{\gamma_{v}}+a_{v} F_{T}^{Z_{v}}\right)-\rho_{T}^{\prime} a_{a} F_{T}^{\prime Z_{a}}\right. \\
& \left.+h\left[\rho_{L} a_{a} F_{L}^{Z_{v}}+\rho_{T} a_{a} F_{T}^{Z_{v}}-\rho_{T}^{\prime}\left(F_{T}^{\prime \gamma_{a}}+a_{v} F_{T}^{\prime Z_{a}}\right)\right]\right\}, \\
= & \frac{\sigma_{M}}{3 \pi^{2} \alpha}\left\{v_{L}\left(F_{L}^{\gamma_{v}}+a_{v} F_{L}^{Z_{v}}\right)+v_{T}\left(F_{T}^{\gamma_{v}}+a_{v} F_{T}^{Z_{v}}\right)-v_{T^{\prime}} a_{a} F_{T}^{\prime Z_{a}}\right. \\
& \left.+h\left[v_{L} a_{a} F_{L}^{Z_{v}}+v_{T} a_{a} F_{T}^{Z_{v}}-v_{T^{\prime}}\left(F_{T}^{\prime \gamma_{a}}+a_{v} F_{T}^{\prime Z_{a}}\right)\right]\right\}
\end{aligned}
$$

where in the second form $(67)$ we have switched to the notation of 6 . The inclusive form factors are defined by

$$
F_{L / T}^{(\prime) C}=\int d \Omega_{n p} f_{L / T}^{(\prime) C}
$$

The expression for the inclusive cross section reflects the well known fact $\|6\|$ that the helicity dependent part is a direct measure of the total parity violating contributions from hadronic parity violation and from electroweak interference. 


\section{HADRONIC PARITY VIOLATION}

A weak parity violating $N N$ interaction $V^{p n c}$ is in principle always present in addition to the strong and parity conserving $N N$ interaction $V^{p c}$, with an estimated relative strength of the order $V^{p n c} / V^{p c} \approx 10^{-7}$. Since the strong $N N$ interaction is highly repulsive at small distances (hard core), the effect of a direct zero range weak $N N$ interaction is negligible. In analogy to the strong $N N$ interaction, one therefore assumes a one-boson-exchange model for the weak $N N$ interaction. In this case, one of the meson-nucleon vertices is parity violating and associated with the weak interaction, while the other (strong) vertex is parity conserving. With this assumption, the whole physics is contained in the weak coupling constants.

Due to the presence of the hard core, one can neglect the exchange of mesons with mass greater than the $\rho$ mass $m_{\rho}$, since they lead to a very short range potential. As shown by Barton 25, the parity violating potential cannot be reduced to one-pion-exchange (OPE) alone, if only strangeness conserving weak currents are included and $C P$ invariance is assumed. This argumentation can be extended to the exchange of any pseudoscalar meson, forbidding the exchange of any neutral pseudoscalar meson (e.g. $\left.\pi^{0}\right)$ and yielding an isovector component $(\Delta I=1)$ in the Hamiltonian for the charged pseudoscalar mesons [1]. Assuming a specific model for the weak interaction, for example, in the GWS-model, one can derive from the transformation properties under isospin rotations that the neutral currents dominate the isovector component of the Hamiltonian [4]. Thus - and due to the fact that in some experiments $\Delta I=1$ is completely absent - the exclusive consideration of OPE (only $\pi^{ \pm}$with $\Delta I=1$ ) is not sufficient and the exchange of vector mesons $(\rho, \omega)$ has to be included. However, the OPE potential is not negligible due to its long range. The resulting effective $N N$ potential is given in Ref. [21]

$$
\begin{aligned}
V^{p n c}(\vec{r}, \vec{p})= & i \frac{f_{\pi} g_{\pi N N}}{2 \sqrt{2} M}\left(\vec{\tau}_{1} \times \vec{\tau}_{2}\right)_{z}\left(\vec{\sigma}_{1}+\vec{\sigma}_{2}\right) \cdot\left[\vec{p}, f_{\pi}(r)\right] \\
& -\frac{g_{\rho}}{M}\left(h_{\rho}^{0} \vec{\tau}_{1} \cdot \vec{\tau}_{2}+\frac{h_{\rho}^{1}}{2}\left(\vec{\tau}_{1}+\vec{\tau}_{2}\right)_{z}+\frac{h_{\rho}^{2}}{2 \sqrt{6}}\left(3 \tau_{1, z} \tau_{2, z}-\vec{\tau}_{1} \cdot \vec{\tau}_{2}\right)\right) \\
& \times\left(\left(\vec{\sigma}_{1}-\vec{\sigma}_{2}\right) \cdot\left\{\vec{p}, f_{\rho}(r)\right\}+i\left(1+\chi_{v}\right)\left(\vec{\sigma}_{1} \times \vec{\sigma}_{2}\right) \cdot\left[\vec{p}, f_{\rho}(r)\right]\right) \\
& -\frac{g_{\omega}}{M}\left(h_{\omega}^{0}+\frac{h_{\omega}^{1}}{2}\left(\vec{\tau}_{1}+\vec{\tau}_{2}\right)_{z}\right) \\
& \times\left(\left(\vec{\sigma}_{1}-\vec{\sigma}_{2}\right) \cdot\left\{\vec{p}, f_{\omega}(r)\right\}+i\left(1+\chi_{s}\right)\left(\vec{\sigma}_{1} \times \vec{\sigma}_{2}\right) \cdot\left[\vec{p}, f_{\omega}(r)\right]\right) \\
& -\frac{g_{\omega} h_{\omega}^{1}-g_{\rho} h_{\rho}^{1}}{2 M}\left(\vec{\tau}_{1}-\vec{\tau}_{2}\right)_{z}\left(\vec{\sigma}_{1}+\vec{\sigma}_{2}\right) \cdot\left\{\vec{p}, f_{\rho}(r)\right\} \\
& -i \frac{g_{\rho} h_{\rho}^{\prime 1}}{2 M}\left(\vec{\tau}_{1} \times \vec{\tau}_{2}\right)_{z}\left(\vec{\sigma}_{1}+\vec{\sigma}_{2}\right) \cdot\left[\vec{p}, f_{\rho}(r)\right]
\end{aligned}
$$

with the usual Yukawa function

$$
f_{\xi}(r)=\frac{e^{-m_{\xi} r}}{4 \pi r}, \quad \text { for } \quad \xi=\pi, \rho, \omega
$$

Furthermore, $M$ denotes the nucleon mass and $\vec{p}=\frac{1}{2}\left(\vec{p}_{1}-\vec{p}_{2}\right)$. Here, seven a priori unknown weak coupling constants appear. Specific values for them are given below.

In order to calculate the opposite parity admixture of the deuteron wave function, perturbation theory of first order is sufficient. The admixed parity violating component is then given by

$$
\left|d_{p n c}^{(1)}\right\rangle=-\frac{1}{H_{p c}^{(0)}-E}\left(H_{p n c}^{(1)}-\left(E-E_{B}\right)\right)\left|d_{p c}^{(0)}\right\rangle,
$$

where $\left|d_{p c}^{(0)}\right\rangle$ is the unperturbed deuteron wave function, $H_{p c}^{(0)}$ and $H_{p n c}^{(1)}$ are the unperturbed strong Hamiltonian and the parity violating perturbation, respectively, $E_{B}$ is the unperturbed deuteron binding energy $(=-2.2246 \mathrm{MeV})$ and $E$ the eigenvalue of the perturbed wave function. The difference $\left(E-E_{B}\right)$ is of second order and, thus, can safely be ignored. In the following calculation, the propagator was approximated by the free Green function. Applying the potential (69) to the unperturbed deuteron wave function yields for the parity violating admixture

$$
\psi_{d}^{p n c}(\vec{p})=\frac{i}{p}\left(\tilde{u}_{11}(p)\left\langle\hat{p} \mid 10 ;(11) 1 m_{d}\right\rangle+\tilde{u}_{10}(p)\left\langle\hat{p} \mid 00 ;(10) 1 m_{d}\right\rangle\right),
$$


where $\tilde{u}_{L S}(p)$ denotes the radial part and $\left\langle\hat{p} \mid T m_{T} ;(L S) J M\right\rangle$ the isospin, spin and angular momentum part of the wave function. In the familiar spectroscopic notation, this corresponds to ${ }^{3} P_{1}$ und ${ }^{1} P_{1}$ states. Both parts obey the Pauli principle, i.e., the wave function is antisymmetric. The radial functions are given by

$$
\begin{aligned}
\tilde{u}_{11}(p)= & -\frac{1}{\pi M \sqrt{3 \pi}} \frac{p}{E_{B}-\frac{p^{2}}{M}} \sum_{L=0,2}(\sqrt{2})^{-L / 2} \int d r j_{1}(p r) \\
& \left\{\frac{f_{\pi} g_{\pi N N}}{\sqrt{2}} e^{-m_{\pi} r}\left(m_{\pi}+\frac{1}{r}\right) u_{L}(r)-g_{\rho} h_{\rho}^{\prime 1} e^{-m_{\rho} r}\left(m_{\rho}+\frac{1}{r}\right) u_{L}(r)\right. \\
& \left.-\left(g_{\omega} h_{\omega}^{1}-g_{\rho} h_{\rho}^{1}\right) e^{-m_{\rho} r}\left[\left(m_{\rho}+(-)^{L / 2} \frac{3}{r}\right) u_{L}(r)-2 u_{L}^{\prime}(r)\right]\right\}
\end{aligned}
$$

and

$$
\begin{aligned}
\tilde{u}_{10}(p)= & \frac{1}{\pi M \sqrt{6 \pi}} \frac{p}{E_{B}-\frac{p^{2}}{M}} \sum_{L=0,2}(-\sqrt{2})^{L / 2} \int d r j_{1}(p r) \\
& \left\{-3 g_{\rho} h_{\rho}^{0} e^{-m_{\rho} r}\left(\left[\left(m_{\rho}+\frac{1}{r}\right) \chi_{v}+\frac{(-2)^{1+L / 2}}{r}\right] u_{L}(r)+2 u_{L}^{\prime}(r)\right)\right. \\
& \left.+g_{\omega} h_{\omega}^{0} e^{-m_{\omega} r}\left(\left[\left(m_{\omega}+\frac{1}{r}\right) \chi_{s}+\frac{(-2)^{1+L / 2}}{r}\right] u_{L}(r)+2 u_{L}^{\prime}(r)\right)\right\} .
\end{aligned}
$$

According to Ref. [26], $h_{\rho}^{\prime 1}$ is comparably small and thus is usually neglected. There exist various approaches to give theoretical estimations for the remaining coupling constants, e.g. [21]227 29]. In Ref. [21] a unified treatment based on the quark model is performed and we use the estimated "best values" given there for the weak coupling constants and listed in Table I.

The radial functions are shown in Fig. 3. For the unperturbed wave function, the parametrization by Machleidt et al. 30] for the Bonn OBEPQ model has been used. In momentum space, the parity violating $P$ waves are much more spread out than the normal $S$ and $D$ waves indicating a much shorter range in coordinate space. This is particularly pronounced in the ${ }^{1} P_{1}$-part because the $\pi$ exchange part of $V^{p n c}$ does not contribute here (see (74)). The total $P$ state probability is $P_{P}=2.7 \times 10^{-14}$, which corresponds to an admixture amplitude that is of the order of magnitude expected [20] and in qualitative agreement with $[15]$.

For the explicit calculation of the structure functions, we have chosen the quasifree region where the total momentum of the exchanged boson can be transferred completely to one nucleon. In this case, the active nucleon is ejected in the forward direction with respect to $\vec{q}$ while the other nucleon acts as a spectator and remains at rest in the lab system. The momentum transfer and the relative $n p$ final energy are then no longer independent. Their dependence defines the quasifree ridge in the $E_{n p}-\vec{q}^{2}$-plane which is characterized by $E_{n p} / \mathrm{MeV} \approx 10 \vec{q}^{2} / \mathrm{fm}^{-2}$. The quasifree kinematics has the advantage that the final state interaction and two-body effects from meson exchange currents are small 31] and thus will be neglected here. This means that we evaluate the $T$-matrix elements of (28) in the Born approximation, taking plane waves as final $n p$ scattering states. Furthermore, as electromagnetic current we use the one-body nucleon current in nonrelativistic reduction to the order $\mathcal{O}(1 / M)$ as is given by

$$
\begin{aligned}
\left\langle N\left(p_{f}, s_{f}\right)\left|\rho^{e . m \cdot}(0)\right| N\left(p_{i}, s_{i}\right)\right\rangle & =\chi_{s_{f}}^{\dagger} G_{E}\left(q^{2}\right) \chi_{s_{i}}, \\
\left\langle N\left(p_{f}, s_{f}\right)\left|\vec{J}^{e . m \cdot}(0)\right| N\left(p_{i}, s_{i}\right)\right\rangle & =\chi_{s_{f}}^{\dagger} \frac{1}{2 M}\left[G_{E}\left(q^{2}\right)\left(\vec{p}_{f}+\vec{p}_{i}\right)+i G_{M}\left(q^{2}\right)(\vec{\sigma} \times \vec{q})\right] \chi_{s_{i}},
\end{aligned}
$$

where $\chi_{s}$ denotes a Pauli spinor, $\vec{q}=\vec{p}_{f}-\vec{p}_{i}$ the momentum transfer, and $G_{E / M}\left(q^{2}\right)$ electric and magnetic Sachs form factors, respectively. We use the dipole parametrization with $G_{E n}$ in the Galster form [32].

In view of the restriction to the one-body current, a remark concerning current conservation is in order. In principle, one has to include in addition also meson exchange current contributions in order to insure gauge invariance. In fact, such meson exchange currents are shown to give a dominant contribution to the nuclear anapole moment [33] in heavy nuclei. But in the case of the deuteron, in particular for the quasifree kinematics we expect that such two-body effects are small, similar to the parity conserving contribution for which this has been shown to be the case [31]. But certainly, it remains to be seen what their effect will be away from the quasifree case.

We show in Fig. 4 for two momentum transfers $\left(\vec{q}^{2}=4 \mathrm{fm}^{-2}\right.$ and $12 \mathrm{fm}^{-2}$, corresponding to $E_{n p}=40 \mathrm{MeV}$ and $120 \mathrm{MeV}$, respectively) the pnc structure functions $f_{T}^{\prime \gamma_{a}}$ and $f_{L T}^{\prime \gamma_{a}}$, which contribute to the helicity dependent part of (65). The transverse structure function $f_{T}^{\prime \gamma_{a}}$ for polarized electrons is of special interest, since its pc equivalent $f_{T}^{\prime \gamma_{v}}$ is absent. As shown in Fig. 田, this structure function has a pronounced minimum at small forward angles and a second 
peak at backward angles. There is no fundamental change for the higher momentum transfer (see right panel of Fig. (4). All in all, this structure function is suppressed by a factor of $10^{-8}$ compared to the pc structure function $f_{T}^{\gamma_{v}}$ [34]. The structure function $f_{L T}^{\prime \gamma_{a}}$ shows a sharp maximum at small forward angles that narrows for the higher momentum transfer but the height remains unchanged. $f_{L T}^{\prime \gamma_{a}}$ is suppressed by roughly $10^{-5}$ compared to the parity conserving structure function $f_{L T}^{\gamma_{v}}$.

With respect to target orientation, we note that in the parity conserving case, the structure functions $f_{L}^{1 M, \gamma_{v}}$, $f_{T}^{1 M, \gamma_{v}}, f_{L T}^{1 M, \gamma_{v}}, f_{T T}^{1 M, \gamma_{v}}, f_{L T}^{\prime 2 M, \gamma_{v}}$ and $f_{T}^{\prime 2 M, \gamma_{v}}$ vanish in Born approximation, as can be seen from (44) through (49), because in this case the corresponding $v$ 's of (53) are real quantities. The nonvanishing parity violating counterparts

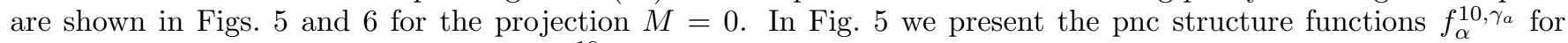
vector polarized deuterons. Except for $f_{T T}^{10, \gamma_{a}}$ they exhibit a typical forward/backward peaking. Finally, the pnc structure functions for a tensor polarized deuteron target are shown in Fig. 6. The largest pnc structure functions for an oriented target are in decreasing order $f_{L T}^{\prime 20, \gamma_{a}}, f_{L}^{10, \gamma_{a}}$ and $f_{T}^{10, \gamma_{a}}$. In general one sees that for a polarized target, the relative order of magnitude between pc and pnc structure functions varies from $10^{-4}$ to $10^{-7}$.

\section{ELECTROWEAK INTERFERENCE}

As we have seen in Sect. II, the presence of the electroweak interference leads to additional structure functions in the expression (38) for the differential cross section. One should keep in mind that the terms proportional to $a_{a}$ result from the interference of the leptonic axial current with the weak hadronic vector current, whereas the structure functions $f_{\alpha}^{(\prime) I M \pm, Z_{a}}$ are a manifestation of the hadronic axial current and thus have a counterpart in $f_{\alpha}^{(\prime) I M \pm, \gamma_{a}}$ resulting from pnc deuteron components.

For the explicit calculation of the structure functions, the same assumptions are made as in Sect. III, i.e., quasifree kinematics and Born approximation. The weak neutral nucleon current consists of a vector and an axial current. The vector current has an analogous form as the e.m. current in Eqs. (75) and (76) but with weak Sachs form factors $\tilde{G}_{E / M}\left(q^{2}\right)$ which are given by

$$
\tilde{G}_{E / M}\left(q^{2}\right)=\frac{1}{2}\left(1-2 \sin ^{2} \theta_{W}\right) \tau_{3} G_{E / M}^{(1)}\left(q^{2}\right)-\sin ^{2} \theta_{W} G_{E / M}^{(0)}\left(q^{2}\right)-\frac{1}{4} G_{E / M}^{(s)}\left(q^{2}\right),
$$

where for $X=E, M$

$$
G_{X}^{(0 / 1)}\left(q^{2}\right)=\frac{1}{2}\left(G_{X, p}\left(q^{2}\right) \pm G_{X, n}\left(q^{2}\right)\right)
$$

denote the isoscalar and isovector e.m. nucleon Sachs form factors and $G_{E / M}^{(s)}\left(q^{2}\right)$ the strangeness form factor, i.e., the contribution of the $s \bar{s}$ density of the nucleon.

The axial current of the nucleon in nonrelativistic reduction is given by

$$
\begin{aligned}
\left\langle N\left(p_{f}, s_{f}\right)\left|\rho^{a}(0)\right| N\left(p_{i}, s_{i}\right)\right\rangle & =\chi_{s_{f}}^{\dagger}\left[\frac{G_{A}\left(q^{2}\right)}{2 M} \vec{\sigma} \cdot\left(\vec{p}_{i}+\vec{p}_{f}\right)\right] \chi_{s_{i}} \\
\left\langle N\left(p_{f}, s_{f}\right)\left|\vec{J}^{a}(0)\right| N\left(p_{i}, s_{i}\right)\right\rangle & =-\chi_{s_{f}}^{\dagger}\left[G_{A}\left(q^{2}\right) \vec{\sigma}\right] \chi_{s_{i}},
\end{aligned}
$$

with the weak axial nucleon form factor

$$
G_{A}\left(q^{2}\right)=-\frac{1}{2} \tau_{3} G_{A}^{(1)}\left(q^{2}\right)+\frac{1}{4} G_{A}^{(s)}\left(q^{2}\right),
$$

where $G_{A}^{(1)}\left(q^{2}\right)$ is defined in (78) for $X=A$. For the axial form factor a dipole parametrization with mass parameter $M_{a}=1.032 \mathrm{GeV}$ is assumed with $G_{A}^{(1)}(0)=1.262$ and $G_{A}^{(s)}(0)=.38$ determined from neutron beta decay and neutrino scattering data, respectively [35]. For the isovector part of the weak vector form factors we use a dipole fit, while for the isoscalar part and the strange form factors we use the fit of Jaffe et al. 36]. We would like to remark that (i) the Eq. (81) is valid at tree level only, because electroweak radiative corrections generate additional isoscalar axial vector components, and (ii) the fit of [36] is not realistic at high momentum transfers [23]. However, in view of the fact, that we do not consider very high momentum transfers and that we are mainly interested in the relative size of electroweak and hadronic parity violation, we do not consider these shortcomings too serious.

Because of the Born approximation, resulting in real $v$ 's, some of the structure functions vanish identically according to (59)-(64). The remaining pnc structure functions from $Z$ exchange without target polarization are shown in Figs. 
7 through 9 for the same kinematics as in Sect. III. Those for the weak hadronic vector current are shown in Figs. 07 and 8, while Fig. 9 refers to the hadronic axial current. Here, all pnc structure functions were multiplied with the appropriate factor $a_{v}$ or $a_{a}$ as they appear in the helicity dependent part of the differential cross section (see $(65))$ in order to represent the appropriate order of magnitude. As has been mentioned, the pnc structure functions from the hadronic vector current are related to the leptonic axial current and thus have no equivalent in the case of $P$-wave admixture in the deuteron. Their size is about $10^{-4}-10^{-7}$ relative to the pc structure functions. Except for $f_{T T}^{Z_{v}}$ at $\vec{q}^{2}=4 \mathrm{fm}^{-2}$, all pnc structure functions exhibit enhanced maxima or minima at forward and backward angles, typical for quasifree kinematics. They show furthermore a stronger dependence on $\vec{q}^{2}$ than the corresponding structure functions for the pnc deuteron components, because for the latter the transition current density is much shorter ranged.

The structure function $f_{T}^{\prime Z_{a}}$ in Fig. 9 is in absolute value about three orders of magnitude higher than its equivalent $f_{T}^{\prime \gamma_{a}}$ due to the $P$-wave admixture. The peaking at forward and backward angles is narrower and for the forward angles of opposite sign (cf. Fig. (4). This is not true for $f_{L T}^{\prime Z_{a}}$ which is of the same order of magnitude, but slightly smaller than the corresponding $P$-wave structure function $f_{L T}^{\prime \gamma_{a}}$ in Fig. 0 . It shows a similar behaviour at forward angles, whereas its minimum at backward angles is much more pronounced.

\section{INCLUSIVE LONGITUDINAL ASYMMETRY}

The parity violating observable that is measured in inclusive electron scattering off an unpolarized deuteron target is the longitudinal asymmetry

$$
\mathcal{A}=\frac{1}{2 h \Sigma_{0}}\left(\Sigma_{+}-\Sigma_{-}\right)
$$

where

$$
\Sigma_{0}=\frac{1}{2}\left(\Sigma_{+}+\Sigma_{-}\right)
$$

With the inclusive differential cross section from (66), $\Sigma_{ \pm}=\Sigma( \pm h)$ for $h>0$, neglecting the tiny contributions from $Z$ exchange, one finds

$$
\Sigma_{0}=c\left(\rho_{L} F_{L}^{\gamma_{v}}+\rho_{T} F_{T}^{\gamma_{v}}\right)
$$

According to (66), one may split the asymmetry into the contributions from hadronic and electroweak parity violation, namely

$$
\mathcal{A}=\mathcal{A}^{\gamma_{a}}+\mathcal{A}^{Z}
$$

where

$$
\begin{aligned}
\mathcal{A}^{\gamma_{a}} & =-\frac{\rho_{T}^{\prime} F_{T}^{\prime \gamma_{a}}}{\rho_{L} F_{L}^{\gamma_{v}}+\rho_{T} F_{T}^{\gamma_{v}}}, \\
\mathcal{A}^{Z} & =\frac{\rho_{L} a_{a} F_{L}^{Z_{v}}+\rho_{T} a_{a} F_{T}^{Z_{v}}-\rho_{T}^{\prime} a_{v} F_{T}^{Z_{a}}}{\rho_{L} F_{L}^{\gamma_{v}}+\rho_{T} F_{T}^{\gamma_{v}}} \\
& =\frac{G_{F}}{2 \sqrt{2}} \frac{q_{\mu}^{2}}{\pi \alpha} \frac{\rho_{L} F_{L}^{Z_{v}}+\rho_{T} F_{T}^{Z_{v}}+\rho_{T}^{\prime}\left(1-4 \sin ^{2} \theta_{W}\right) F_{T}^{\prime Z_{a}}}{\rho_{L} F_{L}^{\gamma_{v}}+\rho_{T} F_{T}^{\gamma_{v}}} .
\end{aligned}
$$

The contributions of the different inclusive form factors are exhibited in Fig. 10 along the quasifree ridge. The largest form factor is the transverse one for the weak hadronic vector current $a_{a} F_{T}^{z_{v}}$. The other two electroweak form factors, $a_{a} F_{L}^{Z_{v}}$ and $a_{v} F_{T}^{\prime Z_{a}}$, reach only one third in magnitude but have the opposite sign than $a_{a} F_{T}^{Z_{v}}$. Three orders of magnitude smaller is $F_{T}^{\prime \gamma_{a}}$, the only form factor from the pnc deuteron components. The resulting longitudinal beam asymmetries $\mathcal{A}^{\gamma_{a}}$ and $\mathcal{A}^{Z}$ are shown in Fig. 11. Since for a given momentum transfer $q^{\text {lab }}$ the asymmetry depends also on the electron kinematics through the lepton density matrices, we have chosen two laboratory scattering angles, one at a more forward direction $\left(\theta_{e}=35^{\circ}\right)$ and one backward angle $\left(\theta_{e}=170^{\circ}\right)$. One readily notes that the beam asymmetry $\mathcal{A}^{\gamma_{a}}$ due to the $P$-wave in the deuteron varies strongly with the scattering angle. Indeed, it is relatively more suppressed for backward scattering than $\mathcal{A}^{Z}$. Furthermore, it is apparent that $\mathcal{A}^{\gamma_{a}}$ is negligible compared to $\mathcal{A}^{Z}$ 
over the whole range of momentum transfers considered here. This means that even for the low momentum transfers of the SAMPLE experiment [13] the contributions from the pnc deuteron components can be neglected.

For the beam asymmetry due to electroweak interference, the dominant contribution comes from the term proportional to $a_{a} F_{T}^{Z_{v}}$, but the dependence on the electron scattering angle is mainly a result of the term proportional to $a_{a} F_{L}^{Z_{v}}$. In order to compare our results on the asymmetry $\mathcal{A}^{Z}$ from electroweak interference with those reported in [16], we show in Fig. 12 the asymmetries on a logarithmic scale. For $\mathcal{A}^{Z}$ we find very good agreement with their results for the quasifree case with plane wave Born approximation.

\section{SUMMARY AND CONCLUSIONS}

In this study two mechanisms of the weak interaction have been investigated that introduce parity violation in electrodisintegration of the deuteron, namely electroweak $\gamma-Z$ interference and parity violating components in the nuclear wave function. We have derived the formal expressions for all observables including beam polarization and target orientation in terms of pc and pnc structure functions. For the explicit calculation, we have concentrated on the quasifree region where final state interaction, meson exchange currents and isobar configurations could be neglected.

In the hadronic part a parity violating $N N$ potential was considered which led to an abnormal parity admixture in the deuteron wave function. The $P$-wave component in the deuteron wave function consists of two parts, a spin singlet and a spin triplet. The total $P$-state probability is very small $\left(2.7 \times 10^{-12 \%}\right)$. Compared to the pc structure functions, the pnc structure functions are suppressed by orders $10^{-4}-10^{-7}$. The resulting parity violating form factors are three orders of magnitude smaller than the ones from $Z$-exchange. For this reason one can safely neglect the contribution of the $P$-wave admixture in the deuteron to the beam asymmetry for longitudinally polarized electrons in the quasifree domain and need only to consider the dominant contribution from $Z$-exchange.

As already mentioned, there is a great deal of interest in experiments to determine strange quark contributions to hadronic matrix elements. Several experiments are underway or planned, e.g., at MIT-Bates [13], Mainz [12] and CEBAF [11]. In connection with this study, the SAMPLE experiment at MIT-Bates [13] is of special interest, since it measures the strange magnetic form factor $G_{M}^{(s)}$ at quite low momentum transfer as determined in parity violating electron scattering off hydrogen and deuterium. Even here, we have not found significant effects from parity violation in the hadronic sector.

In order to study parity violation which originates from the hadronic sector in electromagnetic deuteron break-up, one has to go away from the quasifree kinematics to lower momentum transfer where it may become more comparable in size to the contribution from electroweak interference. But then one has to consider also pnc components in the final state and the contribution from meson exchange currents. In this respect, we consider this study as starting point for further investigations, in particular with respect to the role of final state interaction, meson exchange currents and isobar configurations.

\section{APPENDIX: POLARIZATION OBSERVABLES AND STRUCTURE FUNCTIONS}

In this appendix we will give explicit expressions for the various polarization observables in terms of corresponding structure functions. Denoting a general polarization observable by $X$ as listed in Table II one finds

$$
\begin{aligned}
S_{X}\left(h, P_{1}^{d}, P_{2}^{d}\right)= & P_{X} \frac{d^{3} \sigma^{\gamma+Z}}{d k_{2}^{l a b} d \Omega_{k_{2}}^{\text {lab }} d \Omega_{n p}^{c . m .}} \\
= & \bar{S}_{X}\left(\gamma_{v} ; P_{1}^{d}, P_{2}^{d}\right)+\bar{S}_{X}\left(\gamma_{a} ; P_{1}^{d}, P_{2}^{d}\right) \\
& +a_{v}\left(\bar{S}_{X}\left(Z_{v} ; P_{1}^{d}, P_{2}^{d}\right)+\bar{S}_{X}\left(Z_{a} ; P_{1}^{d}, P_{2}^{d}\right)\right) \\
& +a_{a}\left(\bar{S}_{X}^{\prime}\left(Z_{v} ; P_{1}^{d}, P_{2}^{d}\right)+\bar{S}_{X}^{\prime}\left(Z_{a} ; P_{1}^{d}, P_{2}^{d}\right)\right) \\
& +h\left[\bar{S}_{X}^{\prime}\left(\gamma_{v} ; P_{1}^{d}, P_{2}^{d}\right)+\bar{S}_{X}^{\prime}\left(\gamma_{a} ; P_{1}^{d}, P_{2}^{d}\right)\right. \\
& +a_{v}\left(\bar{S}_{X}^{\prime}\left(Z_{v} ; P_{1}^{d}, P_{2}^{d}\right)+\bar{S}_{X}^{\prime}\left(Z_{a} ; P_{1}^{d}, P_{2}^{d}\right)\right) \\
& \left.+a_{a}\left(\bar{S}_{X}\left(Z_{v} ; P_{1}^{d}, P_{2}^{d}\right)+\bar{S}_{X}\left(Z_{a} ; P_{1}^{d}, P_{2}^{d}\right)\right)\right],
\end{aligned}
$$

where, in analogy to (41), we have defined for $C \in \mathcal{S}_{v} \cup \mathcal{S}_{a}$ 


$$
\begin{aligned}
\bar{S}_{X}\left(C ; P_{1}^{d}, P_{2}^{d}\right)= & c \sum_{I=0}^{2} P_{I}^{d} \sum_{M=0}^{I}\left\{\left(\rho_{L} f_{L}^{I M, C}(X)+\rho_{T} f_{T}^{I M, C}(X)+\rho_{L T} f_{L T}^{I M+, C}(X) \cos \phi\right.\right. \\
& \left.+\rho_{T T} f_{T T}^{I M+, C}(X) \cos 2 \phi\right) \cos \left(M \tilde{\phi}-\bar{\delta}_{I}^{C, X} \frac{\pi}{2}\right) \\
& \left.-\left(\rho_{L T} f_{L T}^{I M-, C}(X) \sin \phi+\rho_{T T} f_{T T}^{I M-, C}(X) \sin 2 \phi\right) \sin \left(M \tilde{\phi}-\bar{\delta}_{I}^{C, X} \frac{\pi}{2}\right)\right\} \\
& \times d_{M 0}^{I}\left(\theta_{d}\right), \\
\bar{S}_{X}^{\prime}\left(C ; P_{1}^{d}, P_{2}^{d}\right)= & c \sum_{I=0}^{2} P_{I}^{d} \sum_{M=0}^{I}\left\{\left(\rho_{T}^{\prime} f_{T}^{\prime I M, C}(X)+\rho_{L T}^{\prime} f_{L T}^{\prime I M-, C}(X) \cos \phi\right) \sin \left(M \tilde{\phi}-\bar{\delta}_{I}^{C, X} \frac{\pi}{2}\right)\right. \\
& \left.\quad+\rho_{L T}^{\prime} f_{L T}^{\prime I M+, C}(X) \sin \phi \cos \left(M \tilde{\phi}-\bar{\delta}_{I}^{C, X} \frac{\pi}{2}\right)\right\} d_{M 0}^{I}\left(\theta_{d}\right),
\end{aligned}
$$

introducing as generalization of (43)

$$
\bar{\delta}_{I}^{C, X}:=\left\{\begin{array}{ll}
\left(\delta_{X, B}-\delta_{I 1}\right)^{2} & \text { for } C \in \mathcal{S}_{v} \\
\left(\delta_{X, A}-\delta_{I 1}\right)^{2} & \text { for } C \in \mathcal{S}_{a}
\end{array}\right\}
$$

The structure functions for a general observable $X$ are given by

$$
\begin{aligned}
f_{L}^{I M, C}(X) & =\frac{2}{1+\delta_{M 0}} \Re\left[i^{\bar{\delta}_{I}^{C, X}} \mathcal{U}_{X}^{00 I M, C}\right] \\
f_{T}^{I M, C}(X) & =\frac{4}{1+\delta_{M 0}} \Re\left[i^{C, X} \mathcal{U}_{X}^{11 I M, C}\right] \\
f_{L T}^{I M \pm, C}(X) & =\frac{4}{1+\delta_{M 0}} \Re\left[i^{\delta_{I}^{C, X}}\left(\mathcal{U}_{X}^{01 I M, C} \pm(-)^{I+M+\delta_{X}, B_{B}+\delta_{C}} \mathcal{U}_{X}^{01 I-M, C}\right)\right], \\
f_{T T}^{I M \pm, C}(X) & =\frac{2}{1+\delta_{M 0}} \Re\left[i^{\delta_{I}^{C, X}}\left(\mathcal{U}_{X}^{-11 I M, C} \pm(-)^{I+M+\delta_{X}, B_{B}+\delta_{C}} \mathcal{U}_{X}^{-11 I-M, C}\right)\right], \\
f_{T}^{\prime I M, C}(X) & =\frac{4}{1+\delta_{M 0}} \Re\left[i^{1+\bar{\delta}_{I}^{C, X}} \mathcal{U}_{X}^{11 I M, C}\right], \\
f_{L T}^{\prime I M \pm, C}(X) & =\frac{4}{1+\delta_{M 0}} \Re\left[i^{1+\bar{\delta}_{I}^{C, X}}\left(\mathcal{U}_{X}^{01 I M, C} \pm(-)^{I+M+\delta_{X}, B}+\delta_{C} \mathcal{U}_{X}^{01 I-M, C}\right)\right],
\end{aligned}
$$

where the $\mathcal{U}_{X}^{\lambda^{\prime} \lambda I M, C}$ are given in terms of the quantities

$$
\begin{aligned}
u_{\lambda^{\prime} \lambda I M}^{s^{\prime} S S \sigma, C}=\frac{\hat{I} \sqrt{3}}{1+\delta_{C, \gamma_{v}}} \sum_{m_{s}^{\prime} m_{s} m^{\prime} m} & (-)^{1-m+s^{\prime}-m_{s}^{\prime}}\left(\begin{array}{ccc}
1 & 1 & I \\
m^{\prime} & -m & M
\end{array}\right)\left(\begin{array}{ccc}
s^{\prime} & s & S \\
m_{s}^{\prime} & -m_{s} & -\sigma
\end{array}\right) \\
\times & \times\left[\left(t_{s^{\prime} m_{s}^{\prime} \lambda m^{\prime}}^{\gamma_{v}}(\theta)\right)^{*} t_{s m_{s} \mu m}^{C}(\theta)+\left(t_{s^{\prime} m_{s}^{\prime} \lambda m^{\prime}}^{C}(\theta)\right)^{*} t_{s m_{s} \mu m}^{\gamma_{v}}(\theta)\right]
\end{aligned}
$$

by the following expressions:

(i) differential cross section $(X=1)$

$$
\mathcal{U}_{1}^{\lambda^{\prime} \lambda I M, C}=\sum_{s} \hat{s} u_{\lambda^{\prime} \lambda I M}^{s s 00, C}
$$

(ii) single nucleon polarization

$$
\begin{aligned}
& \mathcal{U}_{x(1)}^{\lambda^{\prime} \lambda I M, C}=-\sqrt{\frac{3}{2}}\left(V_{\lambda^{\prime} \lambda I M}^{1011, C}-V_{\lambda^{\prime} \lambda I M}^{101-1, C}\right), \\
& \mathcal{U}_{x(2)}^{\lambda^{\prime} \lambda I M, C}=-\sqrt{\frac{3}{2}}\left(V_{\lambda^{\prime} \lambda I M}^{0111, C}-V_{\lambda^{\prime} \lambda I M}^{011-1, C}\right), \\
& \mathcal{U}_{y(1)}^{\lambda^{\prime} \lambda I M, C}=i \sqrt{\frac{3}{2}}\left(V_{\lambda^{\prime} \lambda I M}^{1011, C}+V_{\lambda^{\prime} \lambda I M}^{101-1, C}\right), \\
& \mathcal{U}_{y(2)}^{\lambda^{\prime} \lambda I M, C}=i \sqrt{\frac{3}{2}}\left(V_{\lambda^{\prime} \lambda I M}^{0111, C}+V_{\lambda^{\prime} \lambda I M}^{011-1, C}\right),
\end{aligned}
$$




$$
\begin{aligned}
& \mathcal{U}_{z(1)}^{\lambda^{\prime} \lambda I M, C}=\sqrt{3} V_{\lambda^{\prime} \lambda I M}^{1010, C}, \\
& \mathcal{U}_{z(2)}^{\lambda^{\prime} \lambda I M, C}=\sqrt{3} V_{\lambda^{\prime} \lambda I M}^{0110, C},
\end{aligned}
$$

where

$$
\begin{aligned}
& V_{\lambda^{\prime} \lambda I M}^{101 \sigma, C}=\sqrt{2} \sum_{s^{\prime} s}(-)^{s} \hat{s}^{\prime} \hat{s}\left\{\begin{array}{ccc}
s^{\prime} & s & 1 \\
\frac{1}{2} & \frac{1}{2} & \frac{1}{2}
\end{array}\right\} u_{\lambda^{\prime} \lambda I M}^{s^{\prime} s 1 \sigma, C} \\
& V_{\lambda^{\prime} \lambda I M}^{011 \sigma, C}=\sqrt{2} \sum_{s^{\prime} s}(-)^{s^{\prime} \hat{s}^{\prime} \hat{s}}\left\{\begin{array}{ccc}
s^{\prime} & s & 1 \\
\frac{1}{2} & \frac{1}{2} & \frac{1}{2}
\end{array}\right\} u_{\lambda^{\prime} \lambda I M}^{s^{\prime} s 1 \sigma, C}
\end{aligned}
$$

(iii) double nucleon polarization

$$
\begin{aligned}
& \mathcal{U}_{x x / y y}^{\lambda^{\prime} \lambda I M, C}=-\sqrt{3}\left[V_{\lambda^{\prime} \lambda I M}^{1100, C}+\frac{1}{\sqrt{2}} V_{\lambda^{\prime} \lambda I M}^{1120, C} \mp \frac{\sqrt{3}}{2}\left(V_{\lambda^{\prime} \lambda I M}^{1122, C}+V_{\lambda^{\prime} \lambda I M}^{112-2, C}\right)\right], \\
& \mathcal{U}_{z z}^{\lambda^{\prime} \lambda I M, C}=-\sqrt{3}\left[V_{\lambda^{\prime} \lambda I M}^{1100, C}-\sqrt{2} V_{\lambda^{\prime} \lambda I M}^{1120, C}\right], \\
& \mathcal{U}_{x y / y x}^{\lambda^{\prime} \lambda I M, C}=-\frac{3 i}{2}\left[ \pm \sqrt{2} V_{\lambda^{\prime} \lambda I M}^{1110, C}+\left(V_{\lambda^{\prime} \lambda I M}^{1122, C}-V_{\lambda^{\prime} \lambda I M}^{112-2, C}\right)\right], \\
& \mathcal{U}_{x z / z x}^{\lambda^{\prime} \lambda I M, C}=-\frac{3}{2}\left[ \pm\left(V_{\lambda^{\prime} \lambda I M}^{1111, C}+V_{\lambda^{\prime} \lambda I M}^{111-1, C}\right)+\left(V_{\lambda^{\prime} \lambda I M}^{1121, C}-V_{\lambda^{\prime} \lambda I M}^{112-1, C}\right)\right], \\
& \mathcal{U}_{y z / z y}^{\lambda^{\prime} \lambda I M, C}=\frac{3 i}{2}\left[ \pm\left(V_{\lambda^{\prime} \lambda I M}^{1111, C}-V_{\lambda^{\prime} \lambda I M}^{111-1, C}\right)+\left(V_{\lambda^{\prime} \lambda I M}^{1121, C}+V_{\lambda^{\prime} \lambda I M}^{112-1, C}\right)\right] .
\end{aligned}
$$

where the quantities $V_{\lambda^{\prime} \lambda I M}^{\tau^{\prime} \tau S \sigma, C}$ are given by

$$
V_{\lambda^{\prime} \lambda I M}^{\tau^{\prime} \tau S \sigma, C}=2 \hat{S} \sum_{s^{\prime} s} \hat{s}^{\prime} \hat{s}\left\{\begin{array}{ccc}
\frac{1}{2} & \frac{1}{2} & \tau^{\prime} \\
\frac{1}{2} & \frac{1}{2} & \tau \\
s^{\prime} & s & S
\end{array}\right\} u_{\lambda^{\prime} \lambda I M}^{s^{\prime} s S \sigma, C} .
$$

Note, that the components of the spin operators of both particles refer to the frame associated with the final $n p$ c.m. system denoted by $(x, y, z)$. Its $z$-axis is parallel to $\vec{p}_{n p}$ in the reaction plane and its $y$-axis parallel to $\vec{q} \times \vec{p}_{n p}$ perpendicular to the reaction plane. Thus the polarization components of particle "1" (here the proton) are chosen according to the Madison convention while for particle "2" (neutron) the $y$ - and $z$-components of $\vec{P}$ have to be reversed in order to comply with this convention. The spherical angles of proton and neutron momenta with respect to the reference frame associated with the reaction plane in the c.m. system are $\theta_{p}^{\text {c.m. }}=\theta, \phi_{p}^{\text {c.m. }}=\phi$ and $\theta_{n}^{\text {c.m. }}=\pi-\theta$, $\phi_{n}^{c . m .}=\phi+\pi$ (see Fig. 2).

[1] E.M. Henley, Ann. Rev. Nucl. Sci. 19 (1969) 367

[2] E. Fischbach, and D. Tadić, Phys. Rep. 6C (1973) 123

[3] M. Gari, Phys. Rep. 6C (1973) 318

[4] E.G. Adelberger, and W.C. Haxton, Ann. Rev. Nucl. Part. Sci. 35 (1985) 501

[5] V.M. Dubovik, S.V. Zenkin, I.T. Obukhovskii, and L.A. Tosunyan, Fiz. Elem. Chastits. At. Yadra 18 (1987) 575 (Sov. J. Part. Nucl. 18 (1987) 244)

[6] M.J. Musolf, T.W. Donnelly, J. Dubach, S.J. Pollock, S. Kowalski, and E.J. Beise, Phys. Rep. 239 (1994) 1

[7] J. Ashman et al., Z. Phys. C 57 (1993) 211

[8] K. Abe et al., Phys. Rev. Lett. 74 (1995) 346; 76 (1995) 25

[9] D. Adams et al., Phys. Lett. B 336 (1994) 125; B 357 (1995) 248; hep-ex/9702005

[10] Proceedings Workshop on Parity Violation in Electron Scattering, eds. E.J. Beise, and R.D. McKeown (World Scientific, Singapore 1990)

[11] J.M. Finn, P.A. Souder, Spokespersons, CEBAF-Proposal 91-010 (1991)

[12] D. von Harrach, Spokesperson, MAMI-Proposal A/1-93 (1993)

[13] E.J. Beise et al., Proc. 9th Amsterdam Miniconference "Electromagnetic Studies of the Deuteron", eds. B.L.G. Bakker, T. Ketel, and H. de Vries (NIKHEF, Amsterdam 1996) p. 112 
[14] W.-Y.P. Hwang, and E.M. Henley, Ann. Phys. (N.Y.) 129 (1980) 47

[15] W.-Y.P. Hwang, E.M. Henley, and G.A. Miller, Ann. Phys. (N.Y.) 137 (1981) 378

[16] E. Hadjimichael, G.I. Poulis, and T.W. Donnelly, Phys. Rev. C 45 (1992) 2666

[17] G.I. Poulis, hep-ph/9610536

[18] G.I. Poulis, report ADP-96-47/T241

[19] B. Mosconi, and P. Ricci, nucl-th/9702050

[20] B.H.J. McKellar, Proc. 3. Int. Conf. High Energy Phys. and Nucl. Structure, ed. S. Devons, Columbia University (1969) 682

[21] B. Desplanques, J.F. Donoghue, and B.R. Holstein, Ann. Phys. (N.Y.) 124 (1980) 449

[22] H. Arenhövel, W. Leidemann, and E.L. Tomusiak, Few-Body Syst. 15 (1993) 109

[23] M.J. Musolf, and T.W. Donnelly, Nucl. Phys. A546 (1992) 509

[24] G. Küster, diploma thesis, University Mainz, 1996

[25] G. Barton, Nuovo Cim. 19 (1961) 512

[26] B.R. Holstein, Phys. Rev. D 23 (1981) 1618

[27] M. Gari, and J.H. Reid, Phys. Lett. B53 (1974) 237

[28] J.G. Körner, G. Kramer, and J. Willrodt, Phys. Lett. B81 (1979) 365

[29] V.M. Dubovik, and S.V. Zenkin, Ann. Phys. (N.Y.) 172 (1986) 100

[30] R. Machleidt, K. Holinde, and C.H. Elster, Phys. Rep. 149 (1987) 1

[31] H. Arenhövel, Nucl. Phys. A384 (1982) 287

[32] S. Galster et al., Nucl. Phys. B 32 (1971) 221

[33] W.C. Haxton, E.M. Henley, and M.J. Musolf, Phys. Rev. Lett. 63 (1989) 949

[34] H. Arenhövel, W. Leidemann, and E.L. Tomusiak, Phys. Rev. C 46 (1992) 455

[35] E.J. Beise, and R.D. McKeown, Comm. Nucl. Part. Phys. 20 (1991) 105

[36] R.L. Jaffe, Phys. Lett. B229 (1989) 275

TABLE I. "Best" values of the Glashow-Weinberg-Salam model parameters for the parity violating $N N$ interaction $V^{p n c}$ from 21] in units of $g_{\pi}=3.8 \times 10^{-8}$.

\begin{tabular}{lccccc}
\hline \hline$f_{\pi}$ & $h_{\rho}^{0}$ & $h_{\rho}^{1}$ & $h_{\rho}^{2}$ & $h_{\omega}^{0}$ & $h_{\omega}^{1}$ \\
12 & -30 & -0.5 & -25 & -5 & -3 \\
\hline \hline
\end{tabular}

TABLE II. Listing of polarization observables $X$ (differential cross section $X=1$, and polarization components of outgoing nucleons), separated into scalars (set $A$ ) and pseudoscalars (set $B$ ).

\begin{tabular}{|c|c|c|c|c|c|c|c|c|}
\hline$A$ & 1 & $y(1)$ & $y(2)$ & $x(1) x(2)$ & $x(1) z(2)$ & $y(1) y(2)$ & $z(1) x(2)$ & $z(1) z(2)$ \\
\hline$B$ & $x(1)$ & $z(1)$ & $x(2)$ & $z(2)$ & $x(1) y(2)$ & $y(1) x(2)$ & $y(1) z(2)$ & $z(1) y(2)$ \\
\hline
\end{tabular}




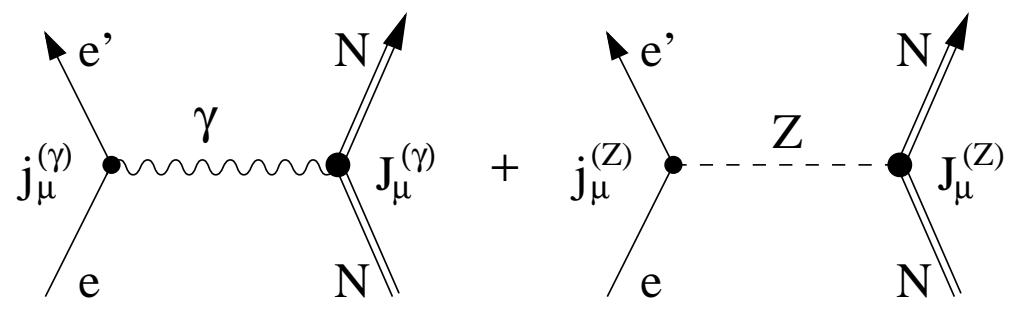

FIG. 1. Diagrams for $\gamma$ and $Z$ exchange in electroweak electron hadron scattering.

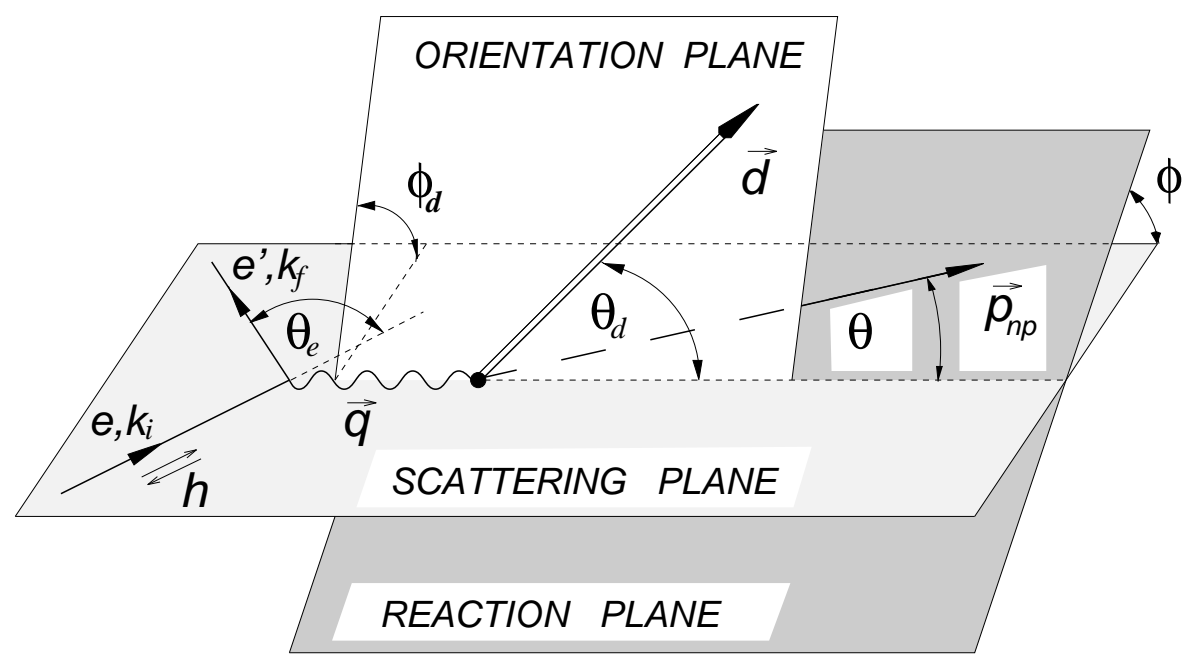

FIG. 2. Geometry of exclusive electroweak deuteron disintegration with polarized electrons and an oriented deuteron target. The relative $n p$ momentum, denoted by $\vec{p}_{n p}$, is characterized by angles $\theta=\theta_{n p}$ and $\phi=\phi_{n p}$ where the deuteron orientation axis, denoted by $\vec{d}$, is specified by angles $\theta_{d}$ and $\phi_{d}$. 

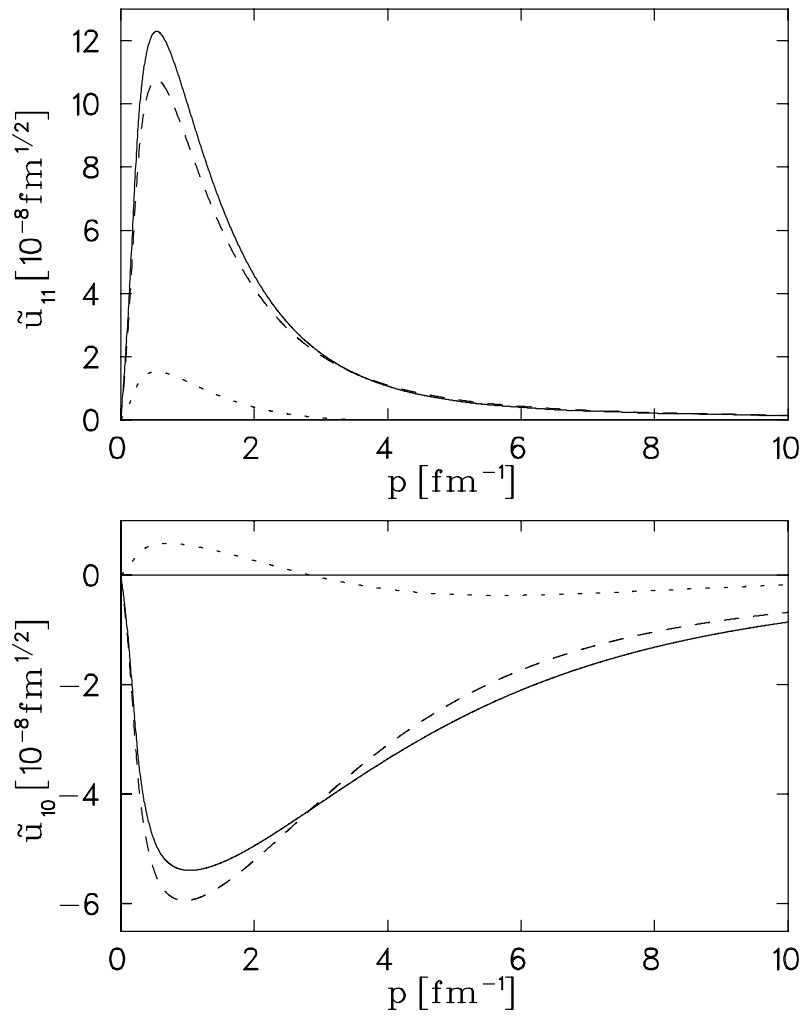

FIG. 3. Radial parts of pnc $P$ wave components (full) of the deuteron wave function in momentum space. Separately shown are the contributions from $S$ (dashed) and $D$ (dotted) components.
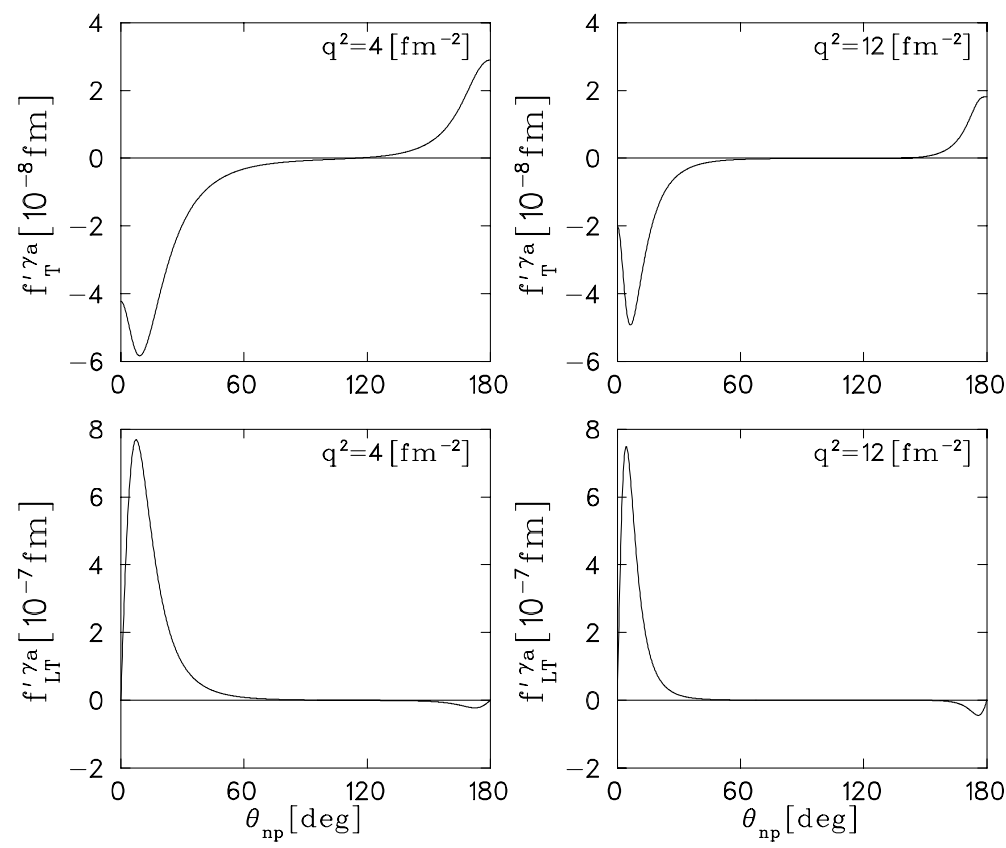

FIG. 4. Pnc structure functions from $P$-wave deuteron components for unoriented target at $\vec{q}^{2}=4$ and $12 \mathrm{fm}{ }^{-2}$. 

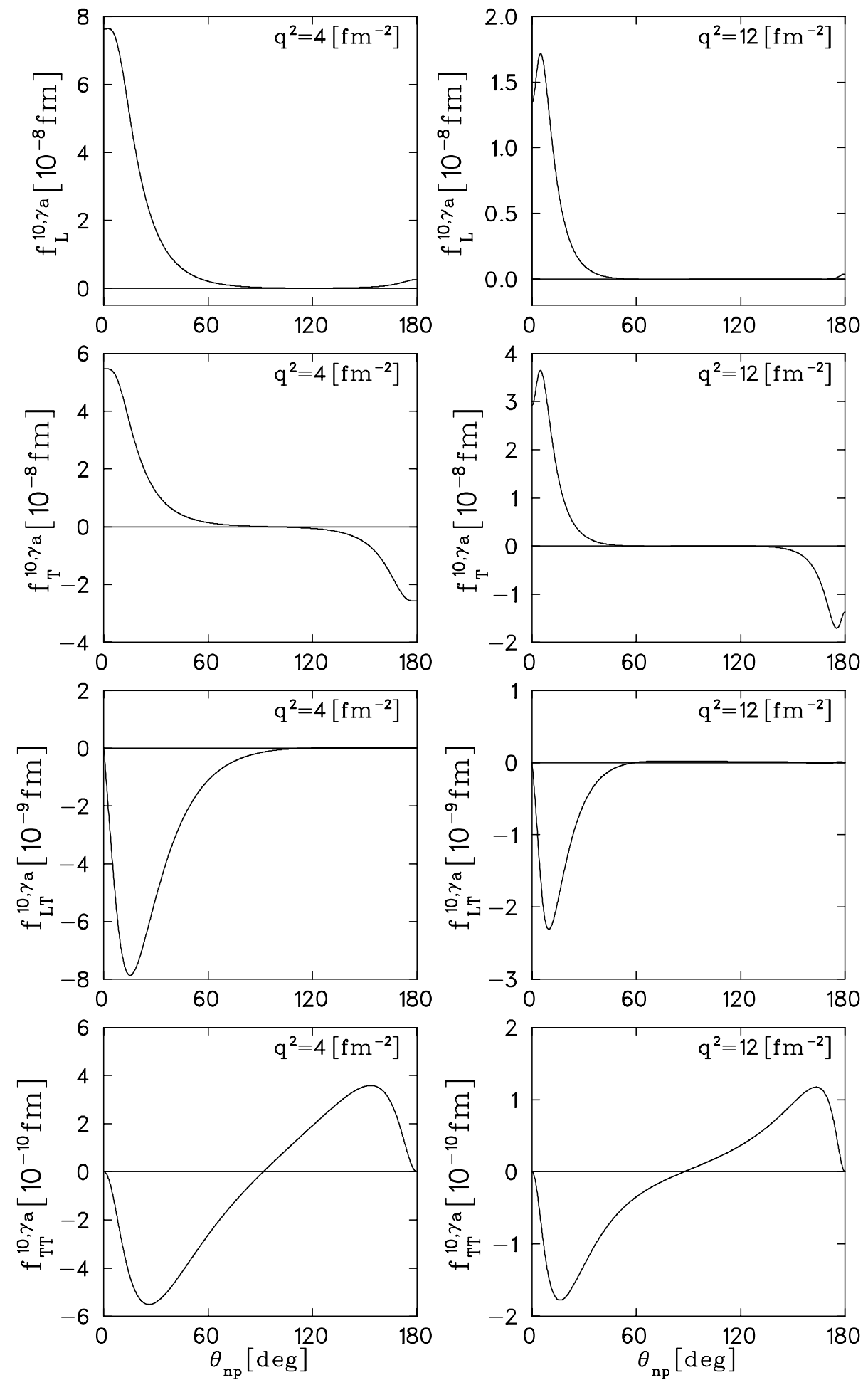

FIG. 5. Pnc structure functions from $P$-wave deuteron components for vector polarized target at $\vec{q}^{2}=4 \mathrm{and}^{2} \mathrm{fm}{ }^{-2}$. 

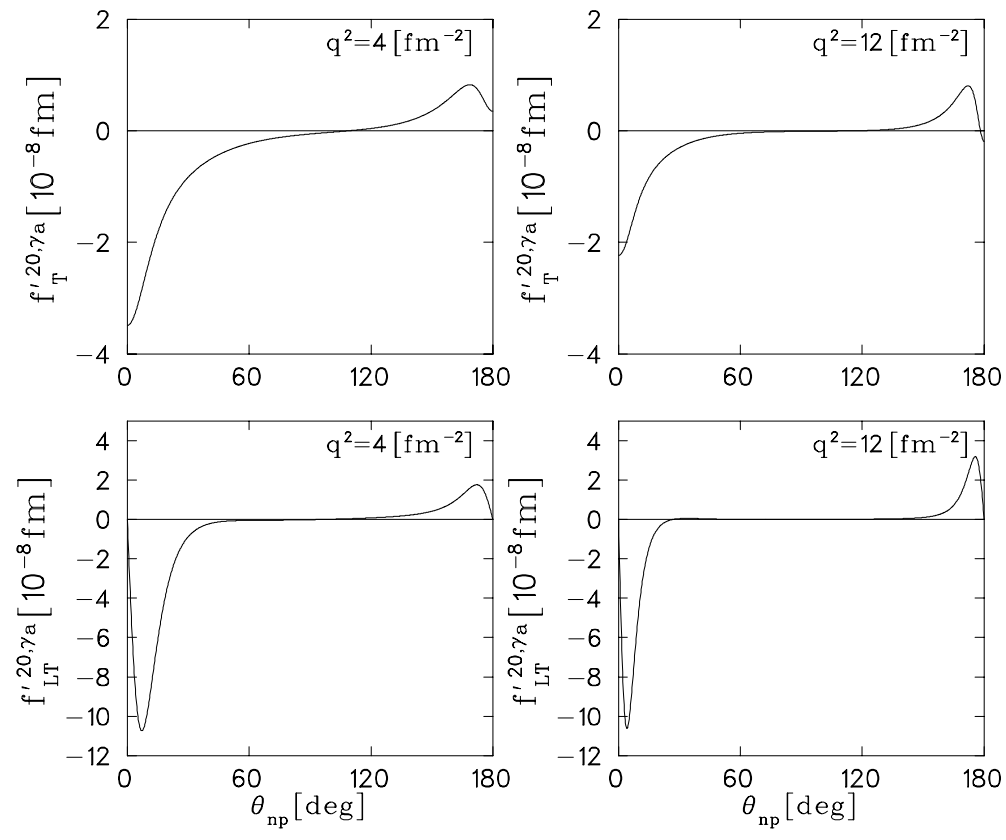

FIG. 6. Pnc structure functions from $P$-wave deuteron components for tensor polarized target at $\vec{q}^{2}=4 \mathrm{and}^{12 \mathrm{fm}}{ }^{-2}$.
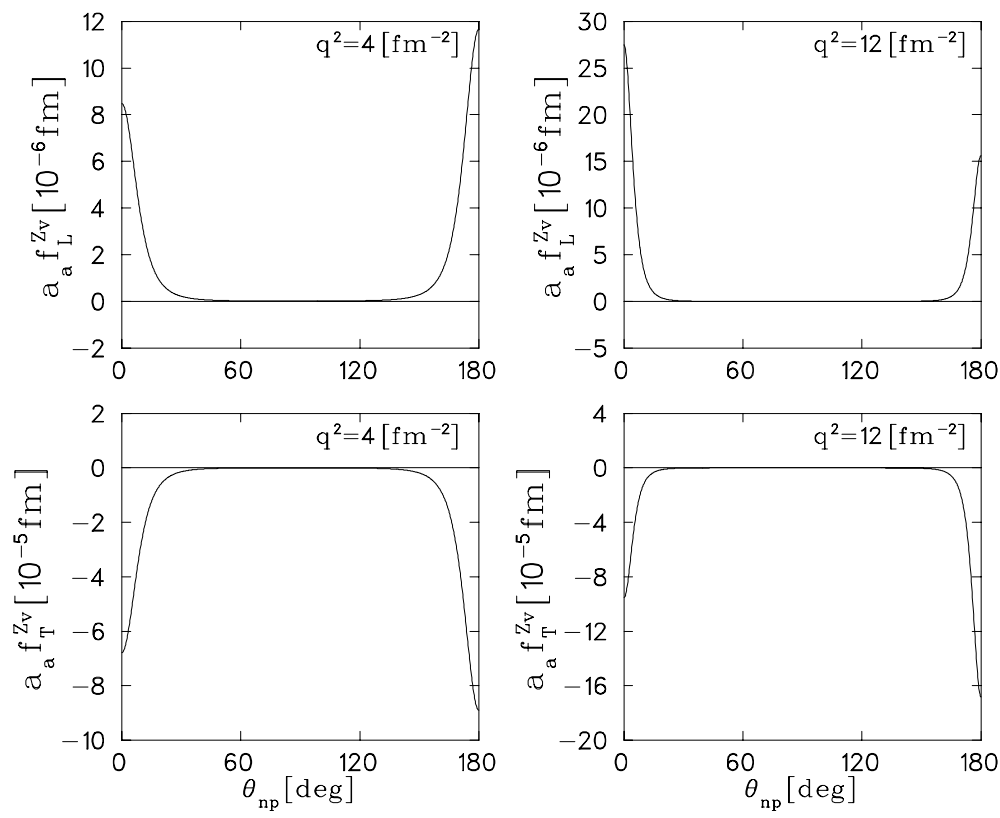

FIG. 7. Longitudinal and transverse pnc structure functions from the weak hadronic vector current at $\vec{q}^{2}=4 \mathrm{and}_{12} \mathrm{fm}{ }^{-2}$. 

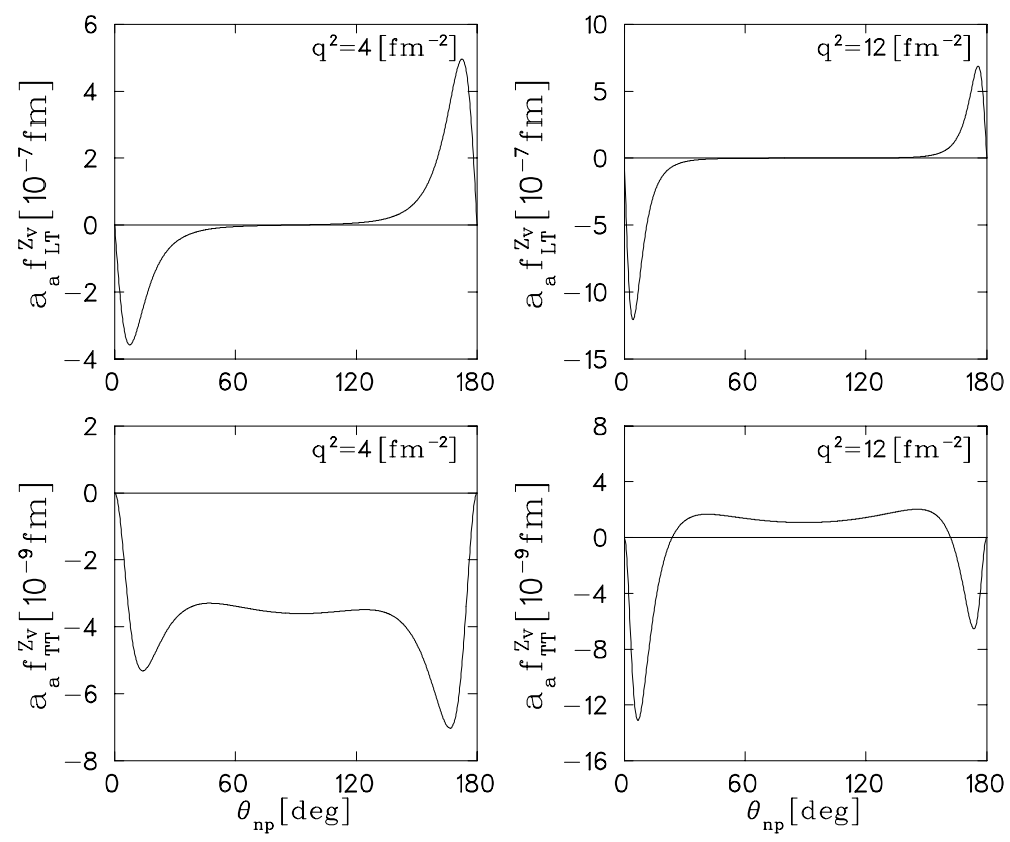

FIG. 8. Longitudinal-transverse and transverse-transverse pnc structure functions from the weak hadronic vector current at $\vec{q}^{2}=4$ and $12 \mathrm{fm}^{-2}$.
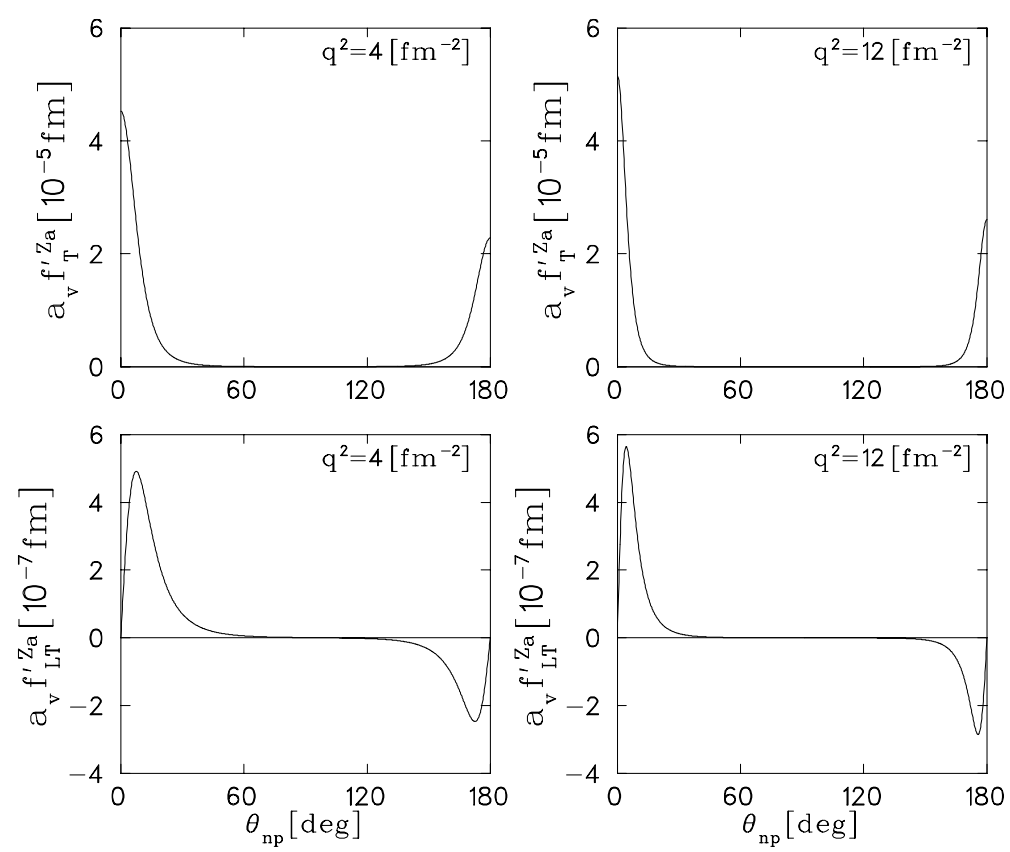

FIG. 9. Transverse and longitudinal-transverse pnc structure functions from the weak hadronic axial current at $\vec{q}^{2}=4$ and $12 \mathrm{fm}^{-2}$. 

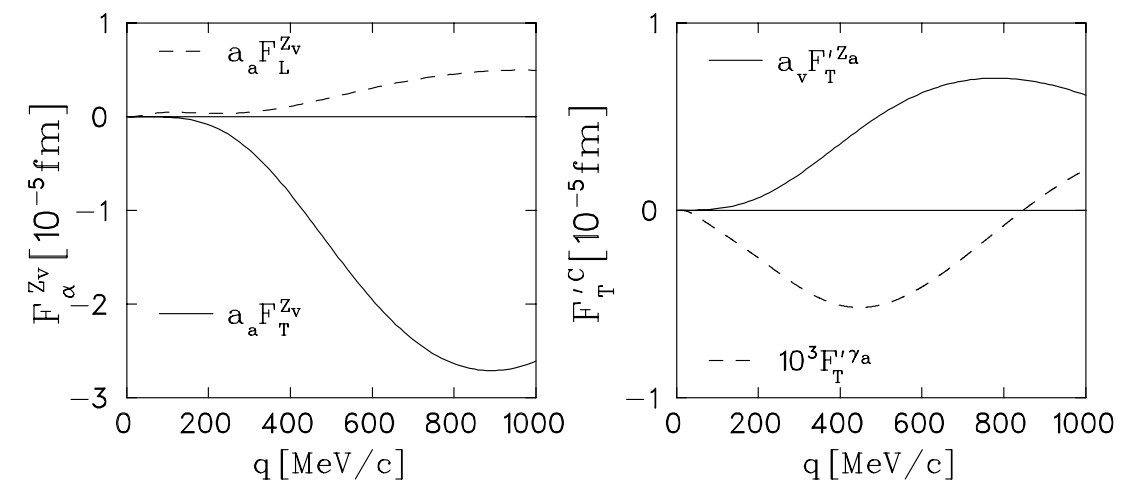

FIG. 10. Pnc form factors along the quasifree ridge for the weak hadronic vector current (left panel), for the weak hadronic axial current and for the pnc deuteron components (right panel).
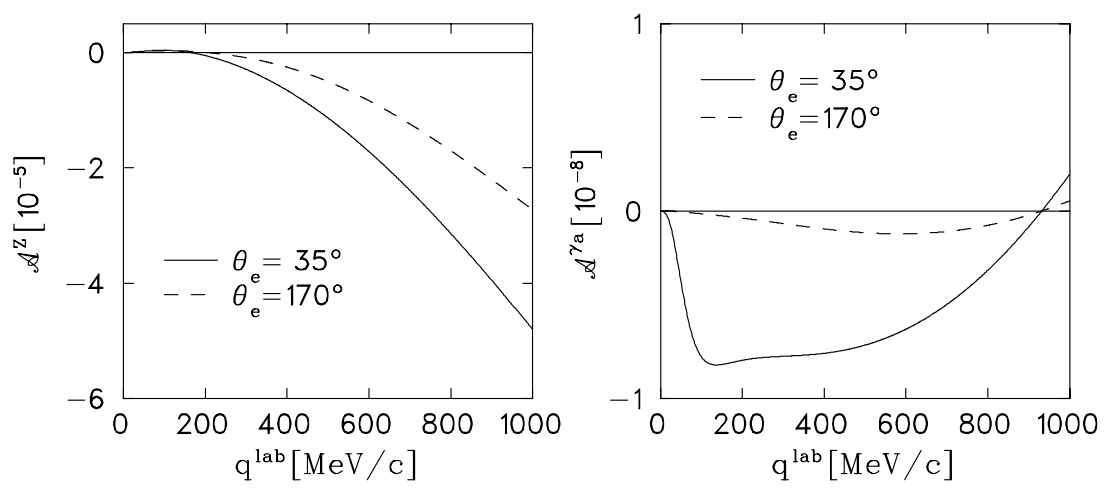

FIG. 11. Contributions to longitudinal asymmetry for polarized electrons along the quasifree ridge from electroweak interference (left panel) and from the pnc deuteron components (right panel) for forward and backward electron scattering $\left(\theta_{e}=35^{\circ}\right.$ and $\left.170^{\circ}\right)$ in the laboratory frame.
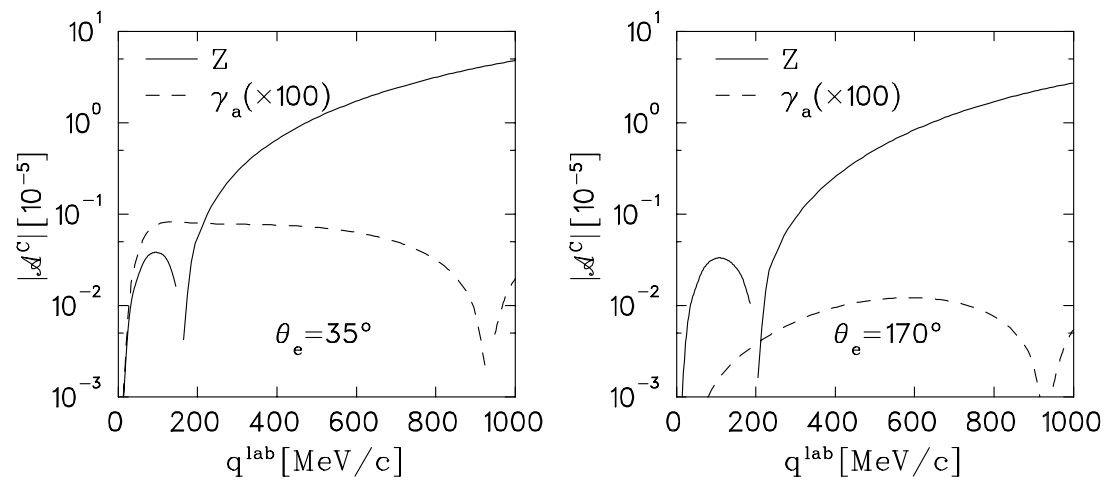

FIG. 12. As Fig. 11 but on a logarithmic scale. 